\title{
ARTICLE
}

\section{KDEL receptor 1 regulates T-cell homeostasis via PP1 that is a key phosphatase for ISR}

\author{
Daisuke Kamimura ${ }^{1,2, \star}$, Kokichi Katsunuma ${ }^{2, \star}$, Yasunobu Arima ${ }^{1,2}$, Toru Atsumi ${ }^{1,2}$, Jing-jing Jiang ${ }^{1,2}$, \\ Hidenori Bando ${ }^{1,2}$, Jie Meng ${ }^{1,2}$, Lavannya Sabharwal ${ }^{1,2}$, Andrea Stofkova1, Naoki Nishikawa1, Hironao Suzuki ${ }^{1,2}$, \\ Hideki Ogura1,2, Naoko Ueda², Mineko Tsuruoka², Masaya Harada², Junya Kobayashi ${ }^{3}$ Takanori Hasegawa4, \\ Hisahiro Yoshida ${ }^{5}$, Haruhiko Koseki ${ }^{4}$, Ikuo Miura ${ }^{6}$, Shigeharu Wakana ${ }^{6}$, Keigo Nishida ${ }^{7}$, Hidemitsu Kitamura ${ }^{7}$, \\ Toshiyuki Fukada7, Toshio Hirano ${ }^{8}$ \& Masaaki Murakami,2
}

KDEL receptors are responsible for retrotransporting endoplasmic reticulum (ER) chaperones from the Golgi complex to the ER. Here we describe a role for KDEL receptor 1 (KDELR1) that involves the regulation of integrated stress responses (ISR) in T cells. Designing and using an $\mathrm{N}$-ethyl- $\mathrm{N}$-nitrosourea (ENU)-mutant mouse line, T-Red (naïve T-cell reduced), we show that a point mutation in KDELR1 is responsible for the reduction in the number of naïve T cells in this model owing to an increase in ISR. Mechanistic analysis shows that KDELR1 directly regulates protein phosphatase 1 (PP1), a key phosphatase for ISR in naïve T cells. T-Red KDELR1 does not associate with PP1, resulting in reduced phosphatase activity against elF2 $\alpha$ and subsequent expression of stress responsive genes including the proapoptotic factor Bim. These results demonstrate that KDELR1 regulates naïve T-cell homeostasis by controlling ISR.

\footnotetext{
${ }^{1}$ Division of Molecular Neuroimmunology, Institute for Genetic Medicine and Graduate School of Medicine, Hokkaido University, Kita-15, Nishi-7, Kita-ku, Sapporo 060-0815, Japan. ${ }^{2}$ Laboratory of Developmental Immunology, Graduate School of Frontier Biosciences, Graduate School of Medicine, and WPI Immunology Frontier Research Center, Osaka University, 2-2, Yamada-oka, Suita 565-0871, Japan. ${ }^{3}$ Radiation Biology Center, Kyoto University, Yoshida-Konoe-cho, Sakyo-ku, Kyoto 606-8501, Japan. ${ }^{4}$ Laboratory for Developmental Genetics, RIKEN Research Center for Allergy and Immunology, 1-7-22 Suehiro-cho, Tsurumi-ku, Yokohama 230-0045, Japan. ${ }^{5}$ Laboratory for Immunogenetics, RIKEN Research Center for Allergy and Immunology, 1-7-22 Suehiro-cho, Tsurumi-ku, Yokohama 230-0045, Japan. ${ }^{6}$ Technology and Development Team for Mouse Phenotype Analysis, RIKEN Bioresource Center, 3-1-1 Koyadai, Tsukuba 305-0074, Japan. ${ }^{7}$ Laboratory for Cytokine Signaling, RIKEN Research Center for Allergy and Immunology, 1-7-22 Suehirocho, Tsurumi-ku, Yokohama 230-0045, Japan. ${ }^{8}$ Osaka University, 2-1, Yamada-oka, Suita 565-0871, Japan. * These authors countributed equally to this work. Correspondence and requests for materials should be addressed to D.K. (email: kamimura@igm.hokudai.ac.jp) or to M.M.

(email: murakami@igm.hokudai.ac.jp).
} 
K DEL receptor 1 (KDELR) was originally found to be responsible for the return of soluble endoplasmic reticulum (ER)-resident proteins to the ER from the intermediate compartment of the cis-Golgi ${ }^{1,2}$. This retrograde transport requires soluble ER-resident proteins to either have a KDEL-like motif at their $\mathrm{C}$ terminus or to form a complex with ER-resident proteins that $\mathrm{do}^{3,4}$. Consistently, it has been reported that KDELR modulates ER stress responses, at least in HeLa cells $^{5}$. A more recent study has suggested that KDELR function goes beyond motif recognition by demonstrating that the chaperone-bound KDELR triggers the activation of Src family kinases at the Golgi complex, a phenomenon that may be critical for intracellular signalling cascades 6,7 .

Integrated stress responses (ISR) are general stress-response programmes conserved from yeast and known to modulate cellular homeostasis by integrating various types of stress signals, including ER stress, amino-acid deprivation, infection with double-stranded RNA viruses, haem deficiency and oxidative stress $^{8-10}$. These diverse signals increase the activation status of four stress kinases-double-stranded RNA-dependent protein kinase R (PKR), RNA-dependent protein kinase-like ER kinase (PERK), eukaryotic initiation factor $2(\mathrm{eIF} 2 \alpha)$ kinase general control non-repressed 2 (GCN2) and haem-regulated eIF2 $\alpha$ kinase, each of which regulates phosphorylation at serine 51 of the $\alpha$ subunit of eIF2. This eIF $2 \alpha$ modification generally attenuates translation, while the activation of ISR via eIF $2 \alpha$ alteration mobilizes the expression of stress-induced genes involved in apoptosis induction, including Bim, CHOP and Trib3 (ref. 11). Furthermore, prolonged phosphorylation of eIF2 $\alpha$ induces apoptosis $^{12,13}$. In particular, protein phosphatase 1 (PP1) interacts with the regulatory proteins GADD34 and CreP to reduce eIF $2 \alpha$ phosphorylation $^{14}$. In addition, defects in ISR are associated with the development of several important pathologies, including diabetes, Alzheimer's disease and viral infection ${ }^{15-17}$. Although ISR can affect the differentiation and activation status of $\mathrm{T}$ cells ${ }^{18,19}$, it remains unknown whether they also play a role in the homeostasis of naïve $\mathrm{T}$ cells in steady state in vivo.

The number of $\mathrm{T}$ cells is relatively constant in the body. When peripheral $\mathrm{T}$ cells are reduced due to thymic involution due to aging, infections or irradiation, the remaining $\mathrm{T}$ cells proliferate to act as a compensatory mechanism for homeostatic proliferation, a response induced by TCR signalling and cytokines ${ }^{20}$. The apoptosis of peripheral $\mathrm{T}$ cells is mainly regulated by $\mathrm{Bim}^{21-24}$, and Bim expression is controlled by Bim transcriptional levels and under stress conditions induced by the transcription factor Chop rather than the forkhead box $\mathrm{O}$ family ${ }^{25}$.

We here establish a new ENU-induced mutant mouse line that shows a decreased number of naïve T-cell numbers, which we name T-Red (naïve T-cell reduced). T-Red mice have a point mutation in KDELR1, and dysfunctional KDELR1 is responsible for the reduction of naïve $\mathrm{T}$ cells. $\mathrm{T}$-cell-mediated responses are attenuated in T-Red mice, and mechanistic analysis suggests that KDELR1 regulates ISR in naïve $T$ cells via PP1 activity. Phosphorylation of eIF $2 \alpha$ is enhanced due to a reduction in activity of PP1 in T-Red naïve T cells, and T-Red KDELR1 does not associate with PP1. Indeed, naïve $\mathrm{T}$ cells in T-Red mice increase the targets of the eIF $2 \alpha$ pathway, such as proapoptotic factors like Bim, CHOP and Trib3, to eventually cause apoptosis. Thus, we suggest that KDELR1 regulates ISR by controlling PP1 activity in naïve $\mathrm{T}$ cells in vivo.

\section{Results}

A mutant strain with excess memory/activated T cells. A mouse library with random genome-wide point mutations was generated by treating $\mathrm{C} 57 \mathrm{BL} / 6$ male mice with the chemical mutagen ethylnitrosourea $(\mathrm{ENU})^{26}$. A first-generation male offspring was bred with wild-type (WT) C57BL/6 female mice, and the second generations were intercrossed. In total, 309 offspring from the third-generation pedigrees were screened for T-cell phenotypes in the peripheral blood by a flow cytometer to identify mutants with aberrant $\mathrm{T}$-cell homeostasis in vivo. In one pedigree, several mice exhibited an unusually high percentage of CD44 expression on the cell surface, which represents the memory/activated T-cell phenotype (Fig. 1a). This phenotype was inherited as a simple autosomal recessive trait (Table 1) and was more evident in CD8 $+\mathrm{T}$ cells than in CD4 $+\mathrm{T}$ cells (Fig. 1a).

To distinguish whether it was the number of memory/activated $\mathrm{T}$ cells that increased or the number of naive $\mathrm{T}$ cells that decreased, we counted T-cell numbers in lymphoid organs. The total cell number in the spleen was reduced in ENU-mutant mice (Fig. 1b). More specifically, the number of CD44lowCD62Lhigh naïve $\mathrm{T}$ cells significantly decreased in the spleen (Fig. 1b, Supplementary Fig. 1). Total cell numbers in the thymus, doublepositive (DP) and single-positive (SP) thymocytes were also less in mutant mice (Fig. 1c,d). To investigate a specific blockade point in the ENU-mutant thymus, we performed flow cytometry analysis and found a reduction of positive selecting thymocytes (CD4 + CD8 + CD5lowCD69low) and positive selected thymocytes (CD4 + CD8 + CD5highCD69high), but an increase of dying thymocytes (Bimhigh, Casp3high or Annexin Vhigh in CD4 + CD8 + CD5lowCD69low thymocytes), suggesting that thymocyte development was inhibited during the selection process in mutant mice (Fig. 1e-h). On the other hand, other cell populations, including CD44 high memory/activated phenotype T cells, double-negative (DN) and CD8 + CD3low immature SP (ISP) thymocytes in the thymus and natural killer cells, $\gamma \delta \mathrm{T}$ cells, neutrophils and dendritic cells of the spleen, were unaffected (Fig. 1b,d,i). We also found that the number of naive B cells but not memory B cells was reduced (Fig. 1i). Interestingly, the T-cell phenotypes described above were more evident after the weaning period (after 5-6 weeks; Fig. 1j). In addition, phenotype differences between $\mathrm{T}$ cells from $\mathrm{T}$-Red mice and those from control mice became more apparent with the developmental stage of $\mathrm{T}$ cells, as shown in Fig. 1b,d (DP thymocytes $<\mathrm{SP}$ thymocytes $<$ naïve $\mathrm{T}$ cells). These results demonstrate that the number of T-cell linage cells significantly decreased in T-Red mice after the DP thymocyte stage, which is when thymocytes obtain TCR complex molecules. We hypothesize that the reduction of thymocytes is induced by their longer lifetime in the thymus due to an accumulation of thymocyte social stress. Thus, we concluded that the T-cell phenotype in the periphery of mutant mice is due to fewer naïve $\mathrm{T}$ cells, not more memory/activated $\mathrm{T}$ cells. Because memory/activated $\mathrm{T}$ cells originate from naïve $\mathrm{T}$ cells, it is likely that the near normal number of memory/activated $\mathrm{T}$ cells in the mutants was caused by homeostatic proliferation ${ }^{27,28}$. We therefore named this mutant strain 'T-Red' (naïve T-cell reduced).

A point mutation in Kdelr1 is responsible for the phenotype. By using F2 mice intercrossed between C57BL/6T-Red homozygotes and a WT $\mathrm{C} 3 \mathrm{H} / \mathrm{He}$ strain, the chromosomal location of the genes responsible for the T-cell phenotype in T-Red mice was mapped within an $\sim 100-\mathrm{kb}$ region of chromosome 7, which contains 443 genes (Fig. 2a). Resequencing the mRNA and genomic DNA exons of T-Red mutants within this region revealed a single $\mathrm{T} \rightarrow \mathrm{C}$ nucleotide substitution in a gene identified as the mouse homologue of Kdelr1 (Fig. 2b). This mutation resulted in a Ser $123 \rightarrow$ Pro amino-acid substitution within the fifth transmembrane region of Kdelr1 (Fig. 2c).

To prove whether this point mutation is responsible for the T-cell phenotype in T-Red mice, we performed two 
a

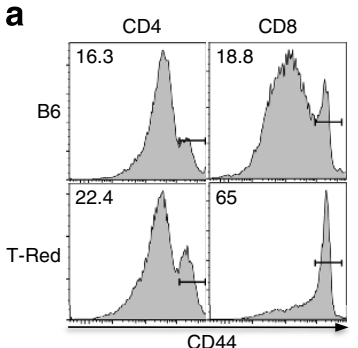

b

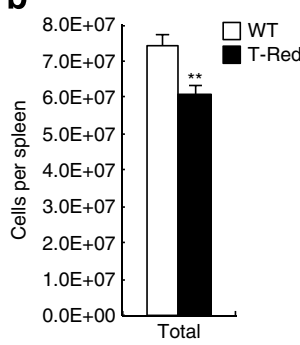

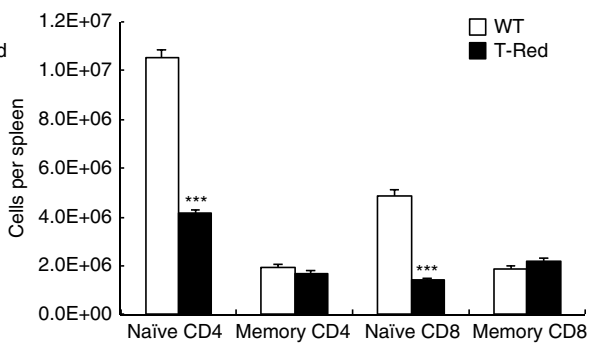

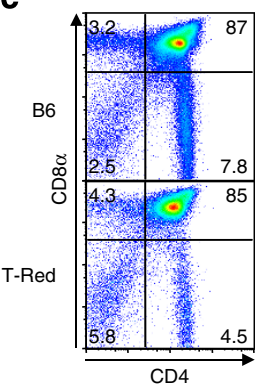

d
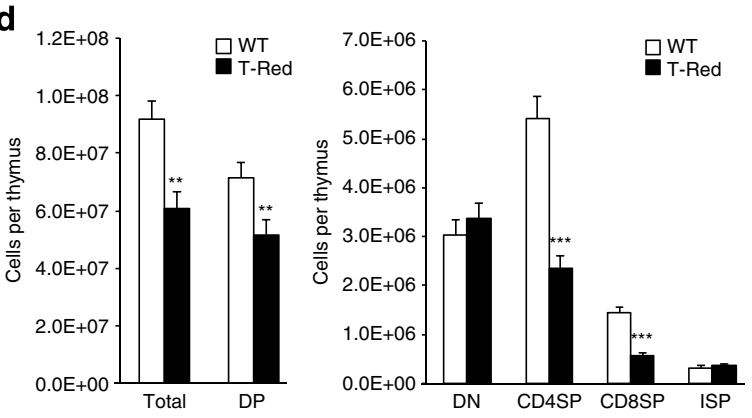

e

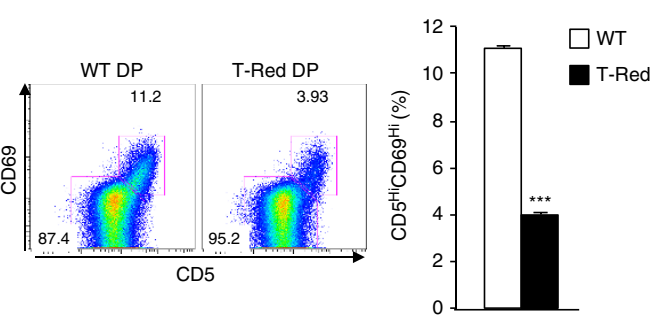

f
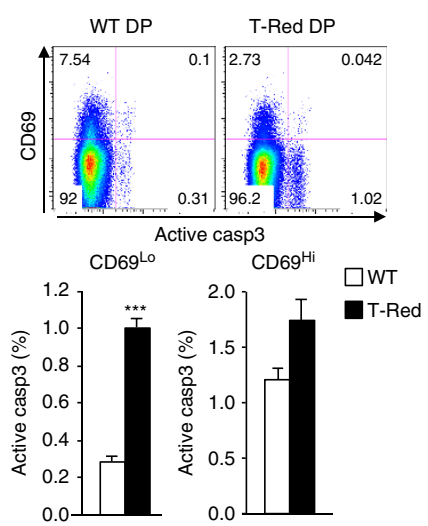

g

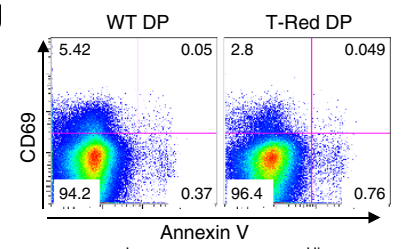

h

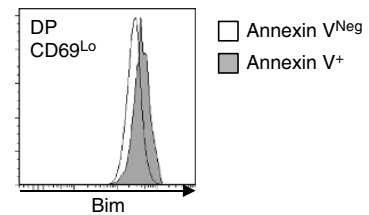

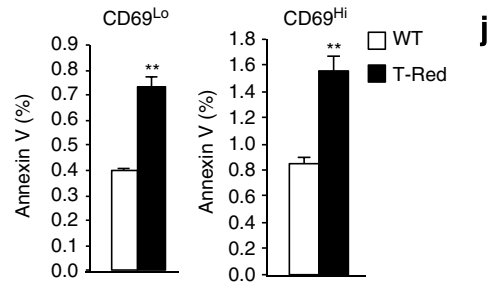
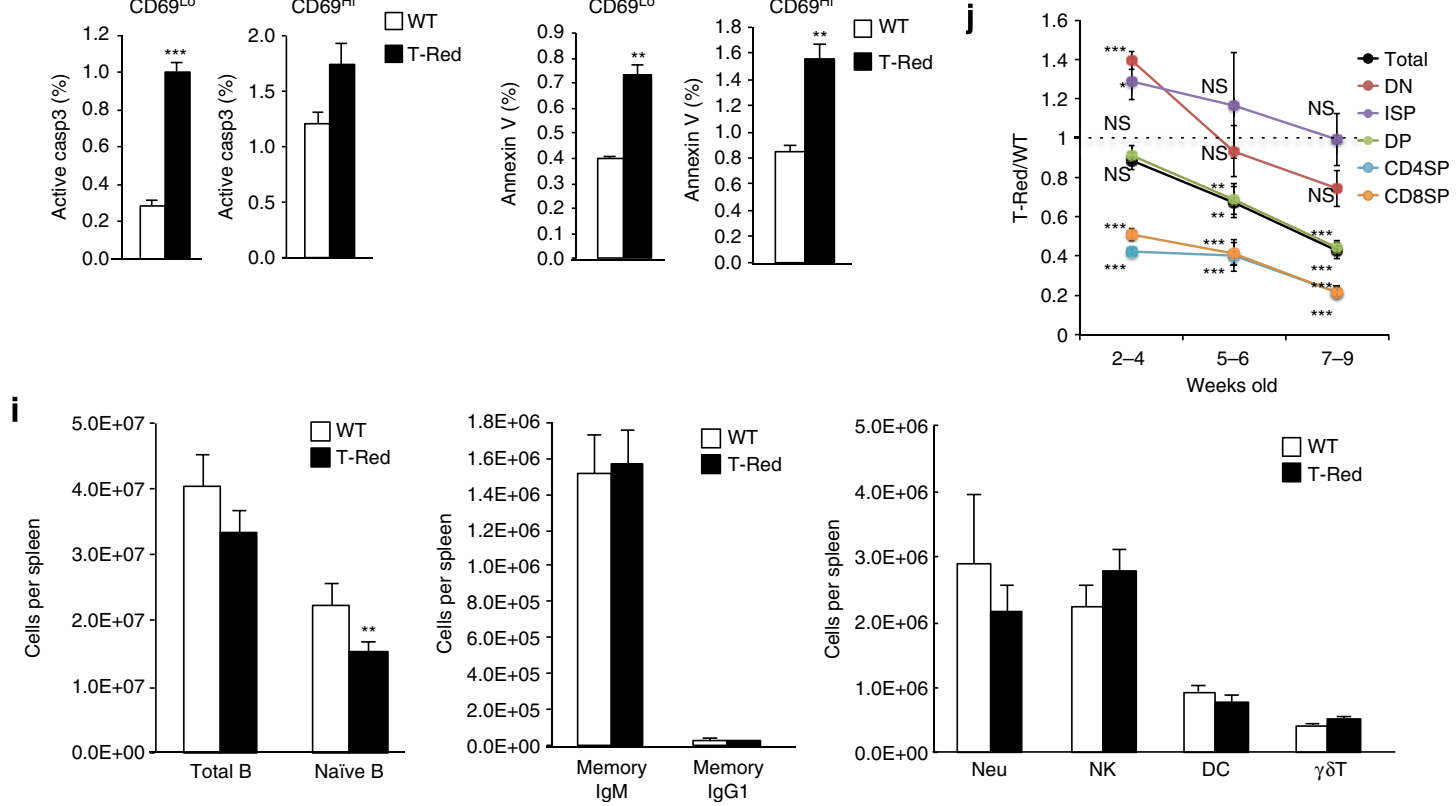

Figure 1 | Establishment of a mutant mouse strain having excess memory T cells. (a) T-cell phenotypes in a mutant strain induced by ENU treatment (T-Red) and control (B6) mice. Flow cytometry analysis was performed using peripheral blood from the mutant strain ( 9 weeks old). Indicated numbers are percentages of memory/activated phenotype T cells, as monitored by CD44 expression. (b) Number of T cells harvested from the spleen. Mice up to 12 weeks old were used. (c) CD4 and CD8 plots of the thymus at 6 weeks old. (d) Number of thymocytes harvested from the thymus. DP, CD4 + CD8 + population; DN (double-negative), CD4-CD8- population; CD4SP (CD4 SP), CD3highCD4 + CD8- population; CD8SP (CD8 SP), CD3highCD4-CD8 + population; and ISP (immature SP), CD3 lowCD4-CD8 + population. Mice up to 12 weeks old were used. (e) CD5 and CD69 levels in CD4 + CD8 + $\mathrm{DP}$ population (left) and the frequency of $\mathrm{CD} 5{ }^{\mathrm{Hi}} \mathrm{CD} 69^{\mathrm{Hi}}$ DP populations (right). Mice at 6-8 weeks old were used. (f,g) The frequencies of active caspase 3 (casp3) (f) and annexin V (g) in DP thymocytes. (h) Bim levels of annexin V-negative (Neg) or positive ( + ) DP CD69Lo thymocytes. (i) Cell numbers of other cell types in the spleen: B (CD19+) cells, naïve B (CD19 + IgM + CD273-) cells, memory lgM (CD19+ IgM + CD273 + ) cells, memory lgG1 $(C D 19+\operatorname{lgG1}+\mathrm{CD} 273+)$ cells, natural killer cells $(N K), \gamma \delta$ T cells $(\gamma \delta \mathrm{T})$, neutrophils (Neu) and dendritic cells (DC). Mice up to 12 weeks old were used. (j) Time course of changes in thymic cellularity. The ratio (T-Red/WT) of each thymic population (cell number) is shown. Data represent the mean + s.e.m. (b, $n>40 ; \mathbf{d}, n>30$; e-g, $n=4-6$ mice; $\mathbf{i}, n=8-20$; and $\mathbf{j}, n=6-14$ for each time point). Representative FACS plots from more than three independent experiments are shown (c,e-h). $P$ values are shown or indicated by asterisks $\left({ }^{\star} P<0.05,{ }^{\star \star} P<0.01\right.$ and $\left.{ }^{\star \star \star} P<0.001\right)$; NS, not significant. 


\begin{tabular}{|lcrcc|}
\hline Table 1 | T-Red phenotype is autosomal recessive. \\
Parents & Generation & n & $\begin{array}{c}\text { Memory } \\
\text { phenotype }\end{array}$ & $\%$ \\
\hline G3.mutant $\times$ G3.mutant & G4 & 28 & 28 & 100 \\
B6 $\times$ G3.mutant & F1 & 21 & 0 & 0 \\
& F2 & 106 & 21 & 19.8 \\
\hline
\end{tabular}

T-cell phenotypes in a mutant mouse strain were inherited as a simple autosomal recessive trait in the progeny. Peripheral blood of mice between 6 and 12 weeks old was examined for CD44 levels of CD8 T cells.

experiments-a retrovirus-mediated rescue experiment using the WT Kdelr1 gene and the design and analysis of Kdelr1 knockout mice. Forced expression of the WT Kdelr1 gene in T-Red-derived haematopoietic stem cells followed by bone marrow transplantation (BMT) increased the percentage of naïve $\mathrm{T}$ cells while concomitantly reducing the memory/activated $\mathrm{T}$-cell fraction, as seen by the decreased surface CD44 expression (Fig. 2d). Furthermore, systemic (Kdelr $1^{\Delta \text { flox/Aflox }}$ mice) and T-cell-specific (CD4-Cre/ Kdelr $1^{\text {flox/flox }}$ mice) deletions of the Kdelr1 gene resulted in almost the same T-cell phenotype as that of T-Red mice (Fig. 2e).

We also examined whether the T-Red phenotype corresponds to the physiological function of KDELR1 molecules. We performed several detailed experiments on mice having deletions of the Kdelr1 gene in T cells (Kdelr $1^{\text {flox/flox }}$ mice crossed with CD4-Cre mice). Data from Kdelr1-deficient mice (Supplementary Fig. 2) were very similar to the data from T-Red mice. In addition, we transferred naïve $\mathrm{T}$ cells from $K d e l r 1^{\text {flox/flox }}$-ERT2-Cre mice into WT hosts and deleted Kdelr1 by treatment with tamoxyfen. Both naïve CD4 $+\mathrm{T}$ cells and CD8 $+\mathrm{T}$ cells were reduced after the tamoxyfen administration (Fig. 2f,g). Therefore, we concluded that the T-Red phenotype corresponds to the physiological function of KDELR1 molecules, at least in T cells, and that the T-Red mutation in the Kdelr1 gene is responsible for the T-Red $\mathrm{T}$-cell phenotype and the loss of function of KDELR1 molecules.

$\mathrm{T}$-cell responses are attenuated in T-Red mice. To investigate whether the reduced number of naïve $\mathrm{T}$ cells in T-Red mice has any impact on antigen-specific $\mathrm{T}$-cell responses, we employed four experimental systems in vivo. In collagen-induced arthritis, clinical scores and serum concentrations of interleukin (IL)-17A were significantly decreased in T-Red mice (Fig. 3a,b). Serum concentrations of anti-ovalbumin (OVA) antibodies after immunization with OVA/Alum were also significantly inhibited in the mutant (Fig. 3c,d). In addition, male, but not female, antigen-specific rejection in female mice was attenuated (Fig. 3e,f), and the $\mathrm{CD} 8+\mathrm{T}$-cell response against Listeria monocytogenes-OVA was less in T-Red mice (Fig. 3g). However, when the same numbers of naïve OT-I and T-Red/OT-I CD8 + $\mathrm{T}$ cells, which have OVA-specific TCR due to a rearranged TCR transgene, were transferred, the expansion of these cells in response to $L$. monocytogenes-OVA infection was found equivalent (Fig. 3h). Similarly, in vitro proliferation and Th17 differentiation were not significantly impaired in T-Red naïve $\mathrm{T}$ cells after stimulation with anti-CD3 antibody (Supplementary Fig. 3). We also confirmed that male antigen-specific rejection in female mice was attenuated in mice having T-cell-specific deletions of the Kdelr1 gene (Supplementary Fig. 2e). Thus, antigenspecific $\mathrm{T}$-cell responses were attenuated in T-Red mice, most likely because of reduced naïve T-cell numbers via the functional defect of KDELR1 molecules. While it is possible that a shorter longevity of animals may occur in certain conventional conditions due to a reduction of $\mathrm{T}$ cells, we observed that T-Red mice had normal longevity and no clear abnormalities even with age in the specific pathogen-free conditions.
Pre-rearranged TCR rescues naïve $T$-cell reduction. We found that CD44 levels of T-Red OT-I T cells were significantly reduced compared with T-Red CD8 + T cells but comparable to WT OT-I T cells (Fig. 4a). Therefore, additional lines of T-Red TCR transgenic strains were generated. Again, the percentages and numbers of naïve $\mathrm{T}$ cells did not show any dramatic decrease in P14, OT-I and OT-II TCR transgenic mice under the T-Red background (Fig. $4 \mathrm{a}-\mathrm{c}$ ). We also found that there was a minimum difference between thymic numbers in OT-I transgenic WT and OT-I T-Red mice (Fig. 4d).

We performed BMT experiments using WT and T-Red mice or regular OT-I and T-Red OT-I mice to further explore the link between the pre-rearranged TCR and T-Red phenotype. The BMT experiments showed results similar to those presented above, as we found a smaller T-cell population in T-Red-derived $\mathrm{BM}$ cells but not in WT-derived BM cells (regular or OT-I case; Fig. 4e,f). All these results suggest that the reduction of naïve $\mathrm{T}$ cells in T-Red mice is dependent on an incomplete TCR rearrangement process and/or TCR signal transduction process in some T-cell repertoires in the thymus and in naïve $\mathrm{T}$ cells in the periphery.

TCR rearrangement in T-Red mice is essentially complete. Because T-Red mice with TCR transgenic backgrounds showed normal percentages of naïve $\mathrm{T}$ cells (Fig. 4a-f), we considered whether the functional defect of KDELR1 induces an incomplete TCR rearrangement process to induce the stress that is stimulated by DNA damage responses. Although TCR J $\alpha$ utilization was perturbed in T-Red T cells, with proximal TCR J $\alpha$ fragments from TCR V $\alpha$ being more rearranged than distal ones (Fig. 4g,h), the total amount of rearranged TCR was equivalent according to a $\mathrm{C} \alpha$ probe as well as qPCR of C $\beta$ (Fig. $4 \mathrm{~h}$ ). We also found normal TCR $\beta$ rearrangements, which were induced by DNA segments in a narrower region compared with TCR $\alpha$ segments ${ }^{29,30}$, in DP thymocytes of T-Red mice and showed normal usage of TCR $\beta$ molecules in naïve CD4 + and naïve CD8 $+\mathrm{T}$ cells in T-Red mice (Supplementary Fig. 4). These results strongly suggest that TCR rearrangement in T-Red mice was essentially complete. Therefore, we concluded that the reduction of naïve $\mathrm{T}$ cells in T-Red mice is not dependent on an incomplete TCR rearrangement process. Instead, the reduction of naïve $\mathrm{T}$ cells in T-Red mice may be dependent on a TCR signal transduction process in some T-cell repertoires of the thymus and in naïve $\mathrm{T}$ cells of the periphery.

Bim and apoptosis increased in T-Red naïve $\mathbf{T}$ cells. We next investigated why the number of naïve $\mathrm{T}$ cells in T-Red mice is lower than in control mice. We first considered whether naïve $\mathrm{T}$ cells from $\mathrm{T}$-Red mice undergo higher rates of apoptosis and examined the expression levels of the proapoptotic factor Bim, a major apoptosis inducer in $\mathrm{T}$ cells, particularly in the periphery $^{21-24}$. T-Red mice showed significantly higher expressions of Bim in their naïve $\mathrm{T}$ cells, but not in their memory/activated $\mathrm{T}$ cells or B cells when compared with controls (Fig. 5a-d). Consistent with these differences in Bim quantity between memory/activated CD4 $+\mathrm{T}$ cells and CD8 $+\mathrm{T}$ cells (Fig. 5b), it is known that memory CD8 $+\mathrm{T}$ cells have more and memory CD4 $+\mathrm{T}$ cells fewer Bim molecules than naïve $\mathrm{T}$ cells ${ }^{31}$ and that memory $\mathrm{CD} 8+\mathrm{T}$ cells are resistant to increased expression of Bim in a manner dependent on the expression of Bcl-2 (ref. 32). Indeed, T-Red naïve $\mathrm{T}$ cells, but not memory/activated ones, showed lower survival rates in the presence or absence of IL-7 (Fig. 5e). We also found that Kdelr1 deficiency in naïve $\mathrm{T}$ cells induced more apoptosis in both $\mathrm{CD} 4+$ and CD8 $+\mathrm{T}$ cells in the presence or absence of IL-7 
a

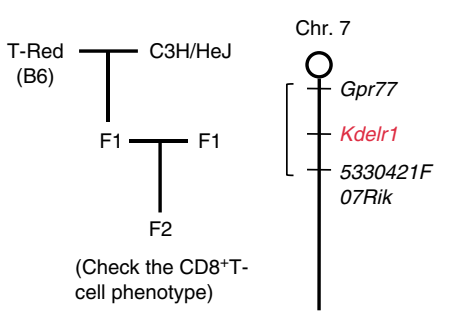

d

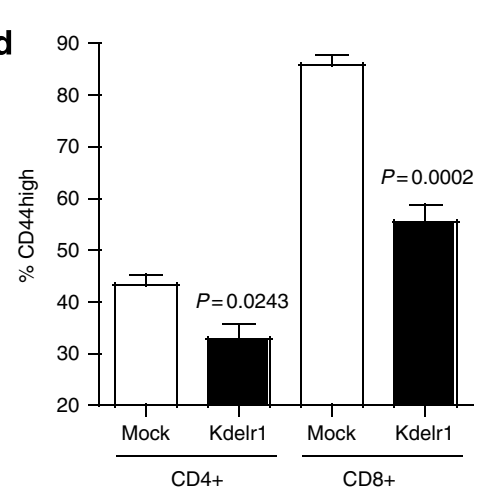

f

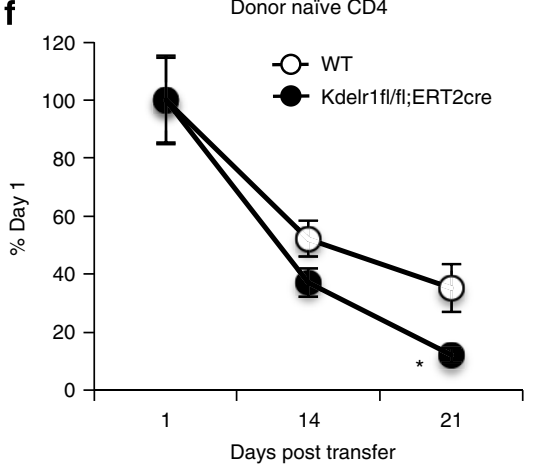

b

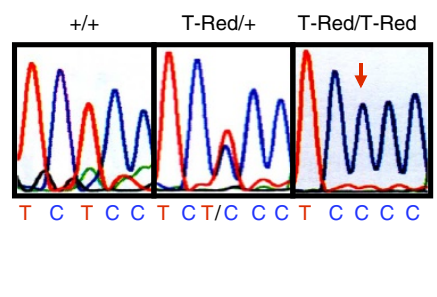

C

WT MNEFRFLGDLSHLLAIILLLIKIWKSRSCAGISGKSQVLFAVVFTARYLDLF NYISLYN T-Red MNLFRFLGDLSHLLAIILLLIKIWKSRSCAGISGESSQVLFAVVFTARYLDLF NYISLYN $* * * * * * * * * * * * * * * * * * * * * * * * * * * * * * * * * * * * * * * * * * * * * * * * * * * * * * * * * * * * * * * *$

WT TCMKVVYIACSFTTVWMIYSKFKATYDGNHDTFR VEFLVVPTAILAFLVNIDFTPLEILW T-Red TCMKVVYIACSFTTVWMIYSKFKATYDGNHDTFRVEFLVVPTAILAFLVNHDFTPLEILW $* * * * * * * * * * * * * * * * * * * * * * * * * * * * * * * * * * * * * * * * * * * * * * * * * * * * * * * * * * * * * * * * *$

WT TFS IYLESVAILPQLFMV KTGEAETITS YLFALGVYRTLYLFNWI IRYHFEGFFDLIA T-Red TFPIYLESVALPQLFMV \$KTGEAETITSHYLFALGVYRTLYLFNWI NRYHFEGFFDLIA **. $* * * * * * * * * * * * * * * * * * * * * * * * * * * * * * * * * * * * * * * * * * * * * * * * * * * * * * * * * *$

WT IVAGLVQTVLYCDFFYLYTTKVLKGKKLSLPA T-Red IVAGLVQTVLYCDFFYLYTTKVLKGKKLSLPA
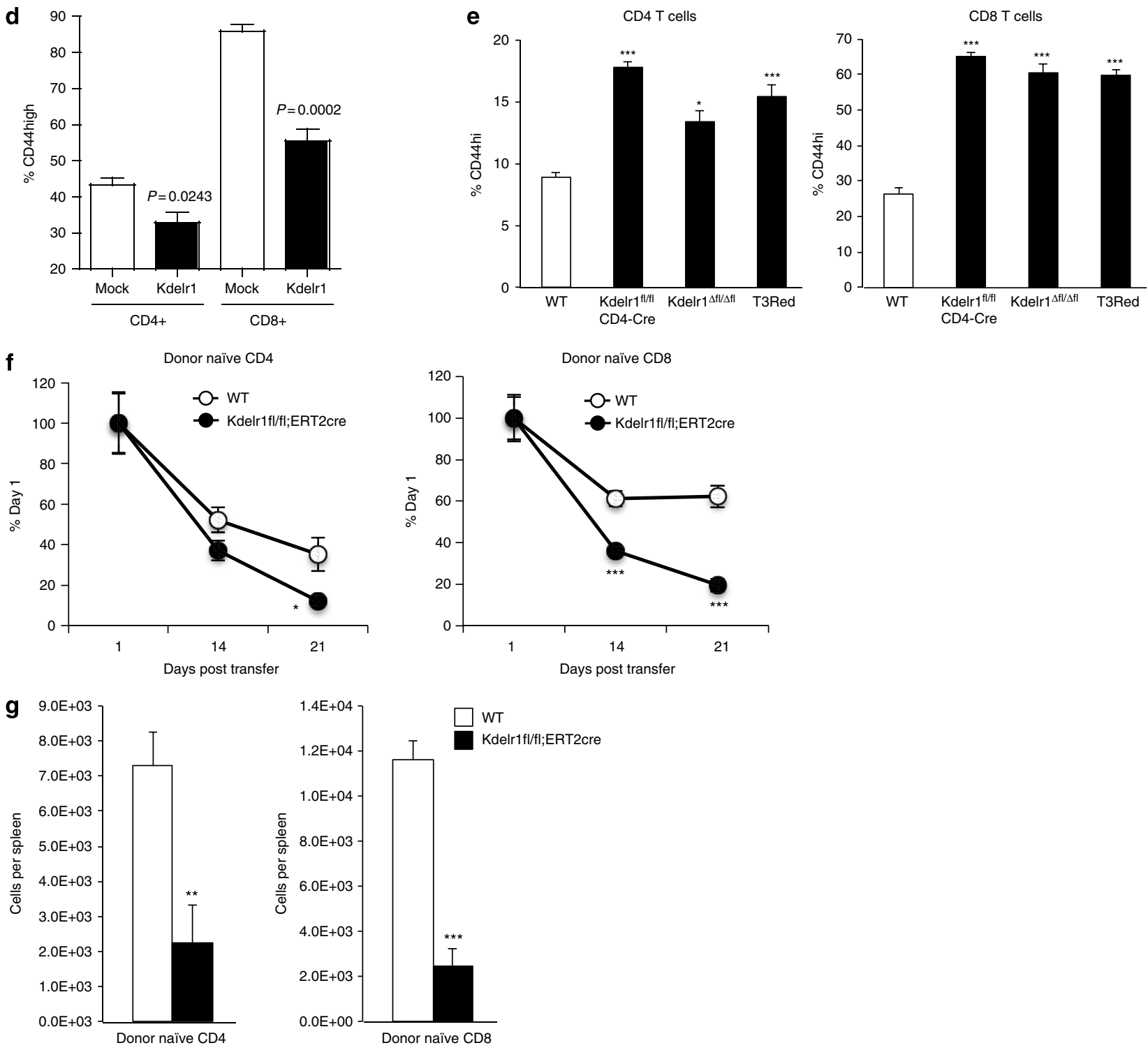

Figure 2 | A point mutation in the Kdelr1 gene is responsible for the T-Red T-cell phenotype. (a) Scheme of the gene mapping (left). The chromosomal location of the gene responsible for T-Red was mapped in an $\sim 100 \mathrm{~kb}$ region between the Gpr77 and 5330421 F07Rik genes of chromosome 7 (right). (b) Resequencing mRNA and genomic DNA exons revealed a single $\mathrm{T} \rightarrow \mathrm{C}$ nucleotide substitution in the Kdelr1 gene mouse homologue. (c) This mutation resulted in a Ser123 $\rightarrow$ Pro amino-acid substitution in the fifth transmembrane domain. Boxes represent presumptive transmembrane domains. (d) Flow cytometry analysis of CD4 + and CD8 + T-cell phenotypes 2 months after retrovirus-mediated forced expression of WT KDELR1 (Kdelr1) or control vector (mock) in T-Red mouse derived haematopoietic stem cells transplanted into the bone marrow (5-6 weeks old). Percentages of CD44 high populations of T cells are shown. Data represent the mean + s.e.m. $(n=3-4)$. (e) Flow cytometery analysis in T cells was performed using peripheral blood from T-Red,

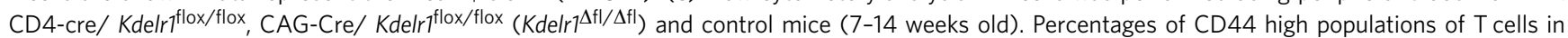
peripheral blood are shown. Data represent the mean + s.e.m. $(n=6-23)$. One-way ANOVA with post hoc Dunnett's test was used. (f) The percentages in

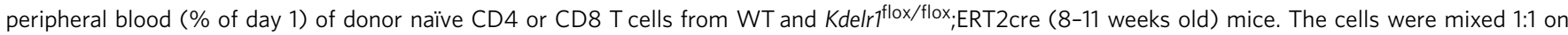
transfer. The host mice were treated with tamoxyfen to induce Kdelr1 deficiency after transfer. ( $\mathbf{g}$ ) The absolute numbers of donor cells in the spleen of the mice in $\mathbf{f}$ on day 21. Data represent the mean + s.e.m. $(n=6)$. $P$ values are shown or indicated by asterisks $\left({ }^{\star} P<0.05,{ }^{\star \star} P<0.01\right.$ and $\left.{ }^{\star \star \star} P<0.001\right)$. 

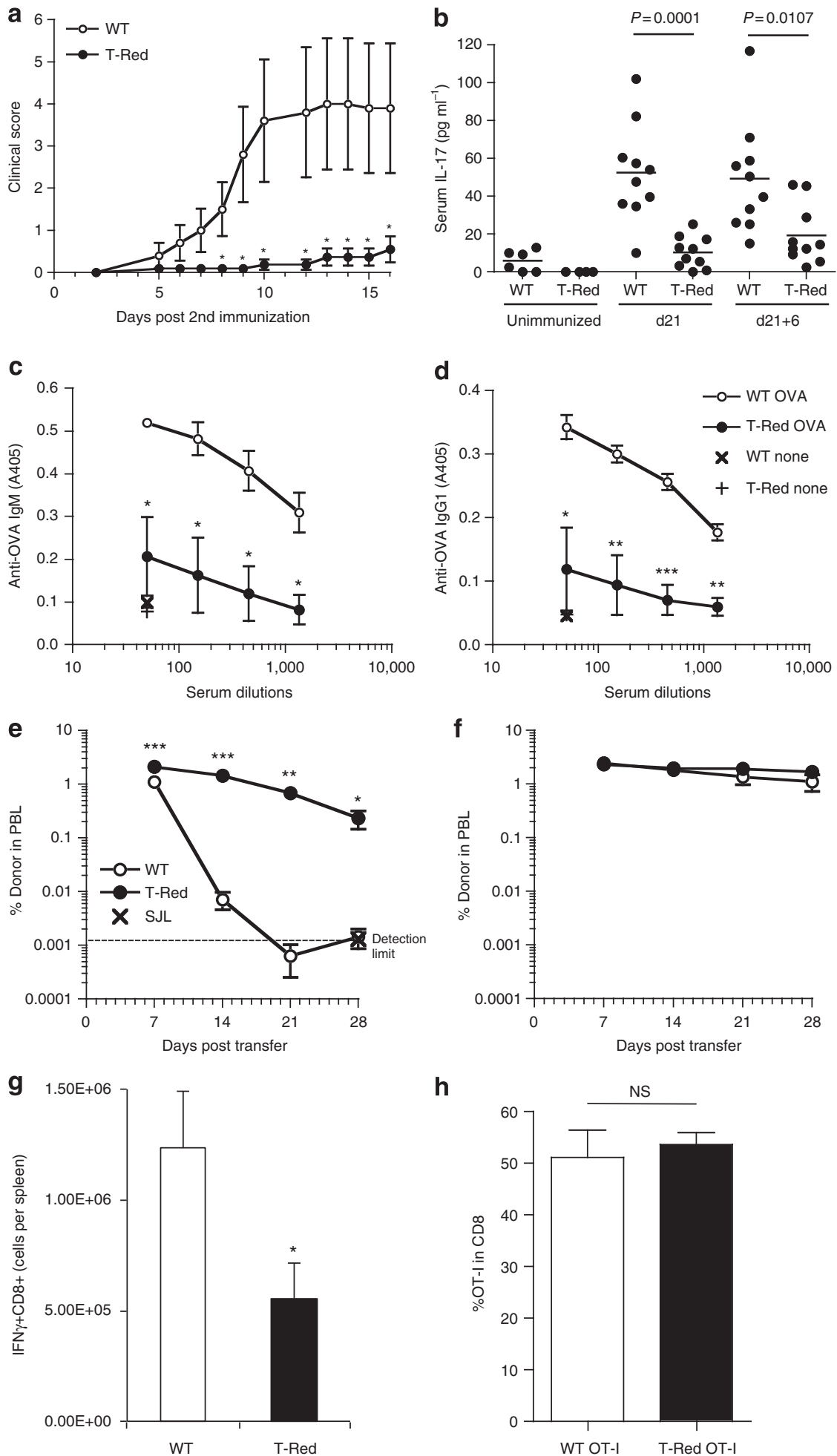

Figure 3 | Antigen-specific T-cell responses were attenuated in T-Red mice. (a,b) Collagen-induced arthritis model. Clinical scores (a) and serum concentrations of IL-17 (b) in T-Red and control mice (5-8 weeks old). Serum IL-17A concentrations were measured by ELISA before antigen immunization (unimmunized), 21 days after immunization (d21) and 6 days after secondary immunization on day $21(\mathrm{~d} 21+6)$. (c,d) T-cell dependent response to OVA. Serum concentrations of the anti-OVA IgM (c) and anti-OVA IgG1 (d) were measured in T-Red and control mice (7-9 weeks old) after immunization with OVA in the presence of alum. (e,f) Male cell rejection in female mice. Congenic CD45.1 male (e) or female (f) splenocytes were transferred into female T-Red or control mice (6-8 weeks old). Percentages of the transferred donor cells in peripheral blood (PBL) are shown. (g,h) Bacteria infection. Listeria monocytogenes expressing OVA was infected in T-Red and control mice (6-8 weeks old). Cell numbers of OVA-specific IFN $\gamma+$ populations in CD8 + T cells on day 7 post infection are shown ( $\mathbf{g})$. The same number (20,000 cells) of OT-I or T-Red/OT-I cells was transferred into WT congenic hosts 1 day before infection. The frequency of donor cells in the blood CD8 + T cells was determined on day 7 post infection (h). Data represent the mean \pm s.e.m. (a, $n=8-10 ; \mathbf{c}-\mathbf{f}, n=4$; and $\mathbf{g}-\mathbf{h}, n=4-5$ ). Wilcoxon test was used in $\mathbf{a} .{ }^{\star} P<0.05 ;{ }^{\star \star} P<0.01 ;{ }^{\star \star \star} P<0.001$. NS, not significant. 
a
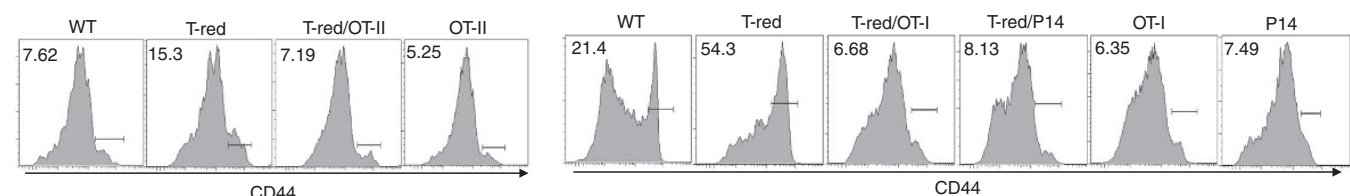

b
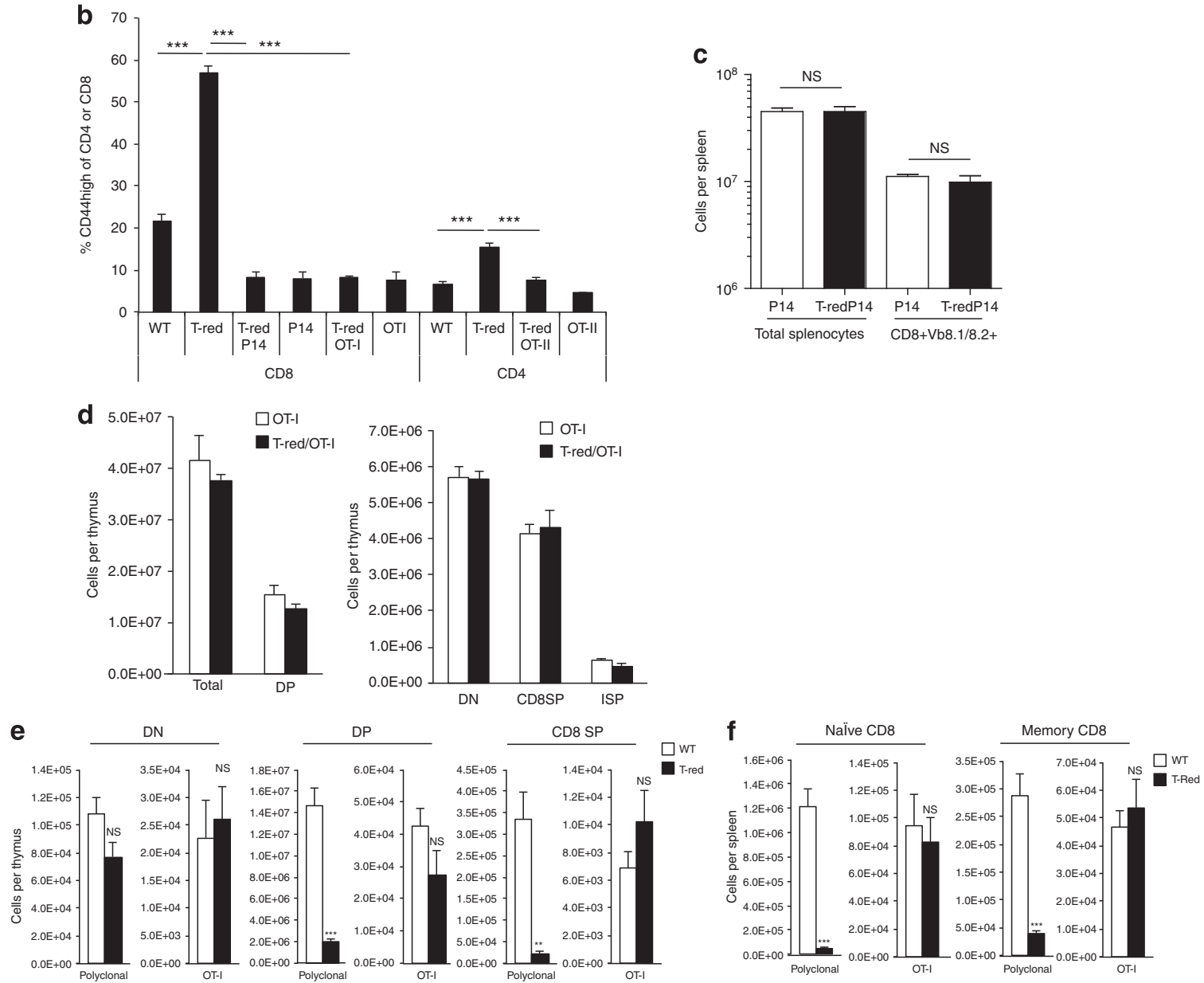

g
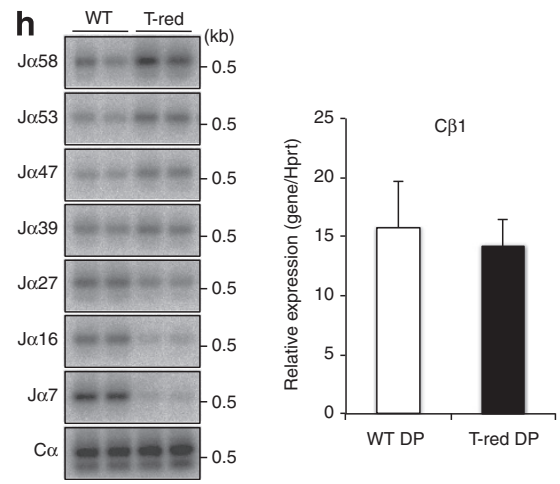

Figure 4 | Pre-rearranged TCR corrected the T-Red phenotype. (a,b) Histograms of CD44 levels (a) and percentages of the CD44 high memory/activated T-cell phenotype (b) in peripheral blood of P14, OT-I and OT-II TCR transgenic mice of T-Red- or WT backgrounds. Mice up to 12 weeks old were used. Representative FACS plots from more than three independent experiments are shown. One-way ANOVA with post hoc Dunnett's test was used in b. (c) Total number of splenocytes and CD8 + V $\beta 8.1 / 8.2+$ T cells in P14 TCR transgenic mice (V $\beta 8.1+$ ) of T-Red or WT backgrounds (6-8 weeks old). (d) Absolute cell numbers of thymocyte subpopulations in OT-I and T-Red/OT-I mice (8-9 weeks old). (e,f) Bone marrow cells from WT (CD45.2/CD90.1), T-Red (CD45.1/CD45.2/CD90.2), OT-I (CD45.2/CD90.1/CD90.2) and T-Red/OT-I (CD45.2/CD90.2) mice were mixed and transplanted into lethally irradiated WT mice (CD45.1/CD90.2; 8-10 weeks old). Each population was tracked by a flow cytometer using congenic markers. Absolute cell numbers of the chimera in the thymus (e) and spleen (f) are shown. ( $\mathbf{g}$ ) Schematic representation of the TCR V $\alpha-J \alpha-C \alpha$ boundary. (h) DP thymocytes were sorted from T-Red and control mice (8-9 weeks old) and isolated from total RNA. RT-PCR was performed using V $\alpha 8-$ and $\mathrm{C} \alpha$-specific primers followed by southern blotting with $\mathrm{J} \alpha$ - or $\mathrm{C} \alpha$-specific probes (left). Representative images from three independent experiments are shown. C $\beta 1$ mRNA levels were examined by quantitative PCR (right). Relative expression levels to Hprt are shown. Data represent the mean + s.e.m. (b, $n=7-39 ; \mathbf{c}, n=4 ; \mathbf{d}, n=4-6 ; \mathbf{e}, \mathbf{f}, n=9 ; \mathbf{h}, n=3$ ). Paired Student's $t$-test was used in $\mathbf{f} .{ }^{\star \star} P<0.01$ and ${ }^{\star \star \star} P<0.001$. NS, not significant. 
(Supplementary Fig. 2d). Furthermore, forced expression of the WT Kdelr1 gene decreased Bim expression in naïve T-Red T cells, but not in memory/activated ones (Fig. 5f). These results suggest that a functional defect in KDELR1 induces the expression of Bim to cause apoptosis in naïve $\mathrm{T}$ cells.

ISR is enhanced in T-Red naïve T cells. We next attempted to identify how the apoptotic pathway is activated in T-Red naive $\mathrm{T}$ cells. We performed DNA microarray analysis using freshly isolated naïve CD4 + and CD8 + T cells from WT and T-Red mice. Because Bim is known to be a target of ISR $^{11}$, we investigated this pathway. Several genes known to be involved in
ISR were also upregulated in T-Red naïve T cells. Quantitative PCR analysis confirmed that ISR-related genes, including Asns, Chop, Trib3 and Vegfa, were significantly upregulated (Fig. 6a). Activated/memory $\mathrm{T}$ cells and B cells from T-Red mice also showed the upregulation of some stress genes, but the induction levels were much lower than in naïve $\mathrm{T}$ cells (Fig. $6 \mathrm{a}$ and Supplementary Fig. 5). In addition, activation of ISR is predicted to globally reduce translation in T-Red cells. Indeed, we found such a reduction in DP thymocytes and naïve $\mathrm{T}$ cells in T-Red mice (Fig. 6b).

A key pathway of ISR is mediated by eIF2 $\alpha$ phosphorylation, which induces cell death when prolonged ${ }^{12,13}$. Phosphorylation of
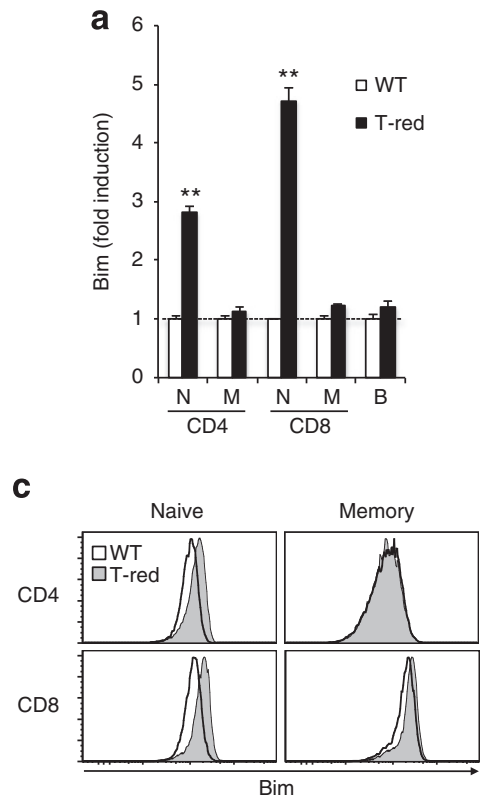

b
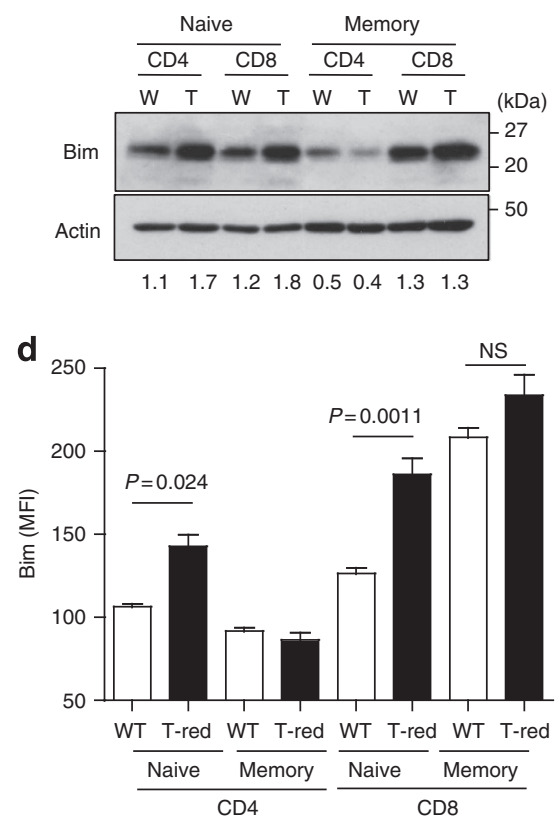
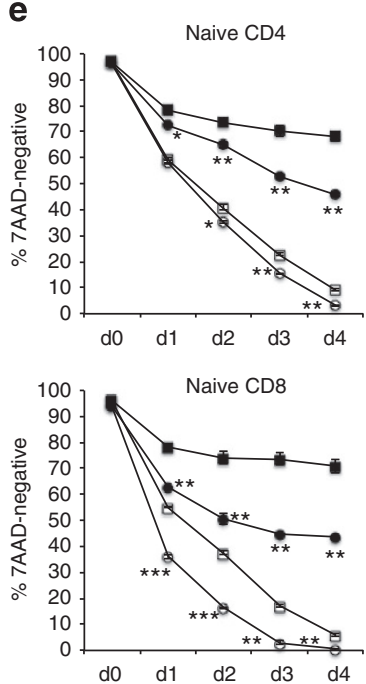
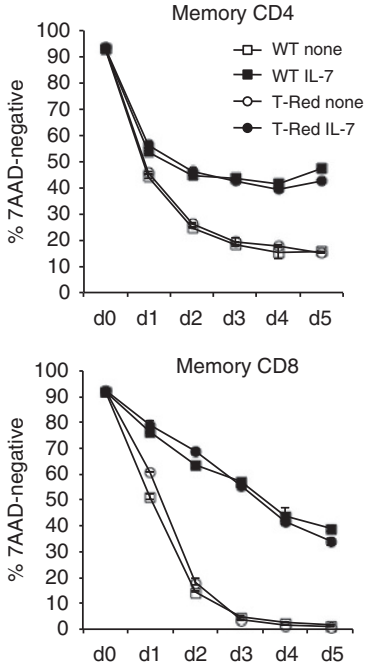

f

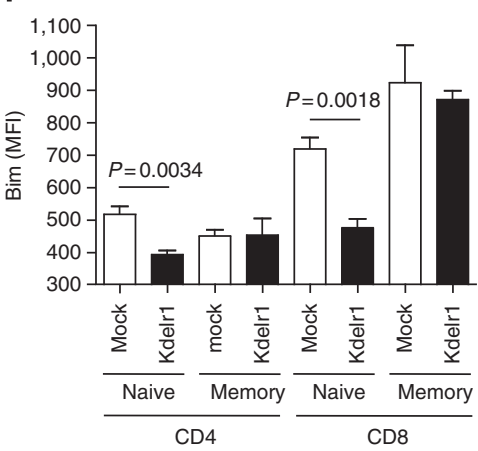

Figure 5 | A functional defect in KDELR1 increases stress-mediated Bim expression and apoptosis in T-Red naïve T cells. (a-d) Bim mRNA and protein levels were investigated by real-time PCR (a), western blot (b) and flow cytometry (c,d) in T-Red (T) and WT (W) mice (8-10 weeks old). $\mathrm{N}, \mathrm{M}$ and $\mathrm{B}$ indicate naïve, memory and B cells, respectively. Expression levels of WT populations were normalized as 1 in $\mathbf{a}$. Numbers in $\mathbf{b}$ represent the intensity ratio of Bim/Actin. Representative images from three independent experiments are shown in $\mathbf{b}, \mathbf{c}$. The mean fluorescence intensity (MFI) of intracellular staining of Bim is shown in $\mathbf{d}(n=3-4)$. (e) In vitro survival of naïve and memory/activated T cells in the presence or absence of IL-7. Mice between 9 and 10 weeks old were used. (f) Bim expression in naïve and memory/activated T cells by flow cytometry following retrovirus-mediated forced expression of WT KDELR1 in T-Red haematopoietic stem cells and BMT $(n=4)$. Mice between 6 and 8 weeks were used. Data represent the mean + s.d. $(\mathbf{a}, \mathbf{e})$ or s.e.m. $(\mathbf{d}, \mathbf{f}) . P$ values are shown in some figures. ${ }^{\star} P<0.05 ;{ }^{\star \star} P<0.01 ;{ }^{\star \star \star} P<0.001$. NS, not significant. 
eIF2 $\alpha$ was monitored by fluorescence-activated cell sorting (FACS) and western blotting and was found to be enhanced in T-Red naïve $\mathrm{T}$ cells (Fig. $6 \mathrm{c}$ and Supplementary Fig. 6a). Importantly, T-Red mice with TCR transgenic backgrounds, which have almost normal numbers of naïve T cells (Fig. $4 \mathrm{a}-\mathrm{c}, \mathrm{f}$ ), significantly decreased the upregulation of ISR-related genes (Fig. 6d). On the other hand, mitochondrial stress, which potentially increases intracellular Bim expression and shortens cell survival time, was not induced in naïve $\mathrm{T}$ cells or thymocytes in T-Red mice, since neither mitochondrial membrane depolarization nor the expression of Clpp and $m t H S P 60$, two target genes of mitochondrial stress ${ }^{33,34}$, were significantly induced (Supplementary Fig. 6b,c). Thus, these results suggest that ISR, but not mitochondrial stress, increased in T-Red naïve $\mathrm{T}$ cells.

Dephosphorylation of eIF2 $\alpha$ by PP1 is impaired. Since phosphorylation of eIF $2 \alpha$ increased in T-Red naïve T cells, we focused on the regulation of eIF2 $\alpha$ phosphorylation to understand how the ISR pathway is activated by KDELR1 dysfunction in T-Red naïve T cells. It is known that four kinases, PKR, PERK, GCN2 and haem-regulated eIF2 $\alpha$ kinase, phosphorylate eIF2 $\alpha$ and that PP1/GADD34 complexes dephosphorylate $\mathrm{it}^{8-10,14}$. Upstream events of the kinase activations, such as cellular ATP (indicative of glucose availability), reactive oxygen species (ROS) and iron levels ${ }^{35-39}$, were not specifically enhanced in $\mathrm{T}$ cells isolated from T-Red mice (Supplementary Fig. 7), suggesting the dephosphorylation of eIF $2 \alpha$ by PP1 may be different. Naïve $\mathrm{T}$ cells showed a certain level of eIF $2 \alpha$ phosphorylation even in WT mice (Fig. 6e). The phosphorylation of eIF $2 \alpha$ was reduced after in vitro culture and reversed by the addition of a PP1/GADD34 specific inhibitor, salubrinal (Fig. 6e $)^{40}$, suggesting that the decline of eIF $2 \alpha$ phosphorylation levels was due to PP1 phosphatase activity. In T-Red naïve T cells, however, phosphorylation of eIF $2 \alpha$ was prolonged, and almost no effect of salubrinal was evident, suggesting a functional defect of PP1/GADD34 phosphatase (Fig. 6e). Importantly, in vitro dephosphorylation assays showed that the phosphatase activity of phospho-eIF2 $\alpha$ was impaired in T-Red naïve CD4 + and CD8 + T cells (Fig. 6f). In addition, only a negligible difference in PP1 defects, which were monitored by the dephosphorylation rates of eIF $2 \alpha$, was detected between WT CD $4+$ and WT CD $8+$ $\mathrm{T}$ cells (Fig. $6 \mathrm{f}$ ). These results suggest that prolonged activation of eIF $2 \alpha$ induced by dysfunctional PP1 phosphatase caused ISR in $\mathrm{T}$-Red naïve $\mathrm{T}$ cells.

Changes in KDELR1 association with PP1. PP1 is known to form complexes with various molecules to determine the PP1 enzyme activity, substrate specificity and subcellular localization $^{41}$. We hypothesized that KDELR1 could be one such partner molecule and therefore investigated the association between KDELR1 and PP1 molecules. As shown in Fig. 6g, PP1 $\alpha$ was co-immunoprecipiated with WT KDELR1. Importantly, the association of PP1 $\alpha$ with the T-Red KDELR1 was substantially weaker than that with WT KDELR1 (Fig. 6g). KDELR1 contains a specific amino-acid sequence, RVEF, which matches a typical PP1-binding motif, $\mathrm{RVXF}^{42}$. However, we found that a loss-of-function mutation in the motif ${ }^{42}$ did not affect KDELR1-PP1 $\alpha$ binding (Fig. 6h). Instead, cytoplasmic loop 1 was involved in the association, but the tail domain, which is known to contribute to the binding of ARF, GAP and Src family kinases $^{6,7,43}$, was not (Fig. 6h). We also determined the region of $\mathrm{PP} 1 \alpha$ responsible for the association. The C-subdomain of $\mathrm{PP} 1 \alpha$, which contains the binding sites for PP1 partners, including Inhibitor-1 and DARPP-32 (ref. 44), was not required for association with KDELR1 (Fig. 6i). Further truncation of PP1 $\alpha$ abolished the association, suggesting that the amino-acid region 182-209 in the C-subdomain is responsible (Fig. 6i). These results support the idea that KDELR1 regulates PP1 activity via direct association.

Moreover, we found that KDELR and PP1 are associated in naïve $\mathrm{T}$ cells and that the degree of association was lower in T-Red naïve CD4 + and naïve CD8 + T cells compared with WT naïve T cells (Fig. 6j). Together with the reduction of T-Red naïve $\mathrm{CD} 4+$ and naïve $\mathrm{CD} 8+\mathrm{T}$ cells, these results suggest that KDELR-PP1 association regulates naïve T-cell survival. Overall, our findings suggest that KDELR-PP1-mediated ISR regulation is involved in peripheral T-cell homeostasis.

\section{Discussion}

It has been long thought that the primary function of KDELR is the retrograde transport of ER chaperones from the Golgi complex to the $\mathrm{ER}^{1,2}$. More recently, it has been found that KDELR also functions by activating Src family kinases on the Golgi complex ${ }^{6,7}$. Here we identify a new role for KDELR that affects naïve T-cell homeostasis in vivo-decreasing ISR-mediated cell death via the direct control of PP1 activity.

We established an ENU-induced mutant mouse strain that has a low number of naïve $\mathrm{T}$ cells ( $\mathrm{T}$-Red mice), finding this phenotype resulted from a point mutation in the Kdelr1 gene. This point mutation caused an amino-acid substitution of KDELR1 at Ser123 to proline (Fig. 2b,c). A previous biochemical study revealed that a mutation at KDELR1 Ser123 abrogates ligand binding, possibly due to conformational alterations ${ }^{45}$. In addition, breeding analysis indicated that the T-Red phenotype is a recessive trait and that mutant mice with Kdelr1 deficiency have a phenotype comparable to T-Red mice (Fig. 2e and Supplementary Fig. 2). Thus, we concluded the S123P point mutation causes dysfunctional KDELR1 in the T cells of T-Red mice and that the T-Red phenotype corresponds to the physiological function of KDELR1 molecules, at least in T cells.

Mechanistic analysis demonstrated that KDELR1 directly regulates PP1 activity, which is critical for preventing ISR in naïve T cells. T-Red KDELR1 association with PP1 was negligible, and its dephosphorylation activity against eIF2 $\alpha$ was diminished in T-Red naïve T cells. In other words, dysfunctional KDELR1 may be unable to regulate eIF2 $\alpha$ activation after the induction of various stresses related to ISR in T cells. It was reported that prolonged activation of eIF $2 \alpha$ leads to apoptosis in cell lines in vitro ${ }^{12}$ and in neurons in vivo ${ }^{13}$. Consistent with these observations, T-Red naïve $\mathrm{T}$ cells showed an upregulation of genes encoding Bim, Chop and Trib3, all target genes and sometimes effector genes of the ISR pathway. Taken together, dysregulation of PP1 by the KDELR1 T-Red mutation induces ISR-mediated upregulation of proapoptotic factors including Bim, which may cause apoptosis in naïve $\mathrm{T}$ cells.

What is the relationship between the TCR-mediated signal and ISR? TCR transgenic data suggest that these transgenic TCR-mediated signals might mainly transduce a positive survival signal in naïve $\mathrm{T}$ cells in a manner dependent on TCR affinity for endogenous peptides (on MHC molecules) at steady state. Therefore, it is possible that a strong TCR signal rescues T-Red naïve $\mathrm{T}$ cells from ISR-mediated apoptosis by enhancing the activation of PP1 followed by the dephosphorylation of eIF $2 \alpha$. On the other hand, we also suggest that the reduction of naïve $\mathrm{T}$ cells is induced by their longer lifetime in the periphery most likely due to an accumulation of T-cell social stress.

Because we found that the numbers of DP and SP cells in T-Red OT-I mice are not reduced (Fig. 4d,e), we also hypothesize that TCR transgenic thymocytes transduced a relatively strong TCR signal from endogenous antigens compared with non- 
transgenic thymocytes just like naïve $\mathrm{T}$ cells are rescued from an excess ISR. Therefore, the transgenic thymocytes were negligibly affected even by the enhanced ISR in T-Red mice due to the excessive survival signal from the transgenic TCR. On the other hand, non-transgenic thymocytes that transduced a low TCR signal might be sensitive to the enhanced ISR in T-Red background, while those cells that have a high TCR signal were not. We, however, believe that the signalling events during the thymic selections are more complex compared with those in peripheral $\mathrm{T}$ cells. Therefore, further investigation is needed to answer this hypothesis particularly by using other high and low affinity TCR transgenic mice.

It remains unclear why proximal TCR $\alpha$ rearrangements were favoured in T-Red cells. One possibility is that proximal TCR $\alpha$ rearrangements make the time length of the DP stage in T-Red mice short to prevent ISR-mediated apoptosis induction, as it was a

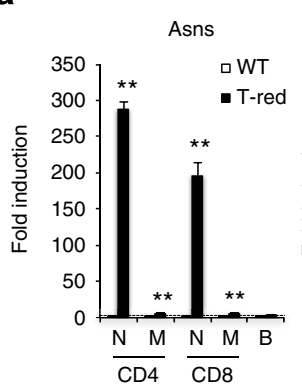

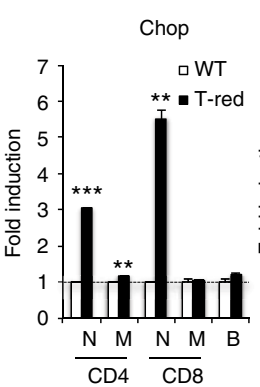

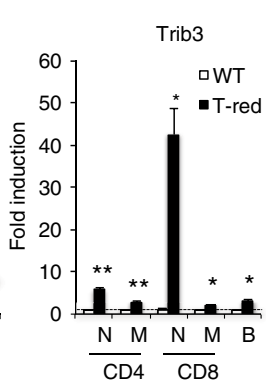

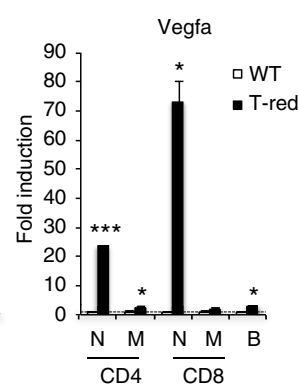

b
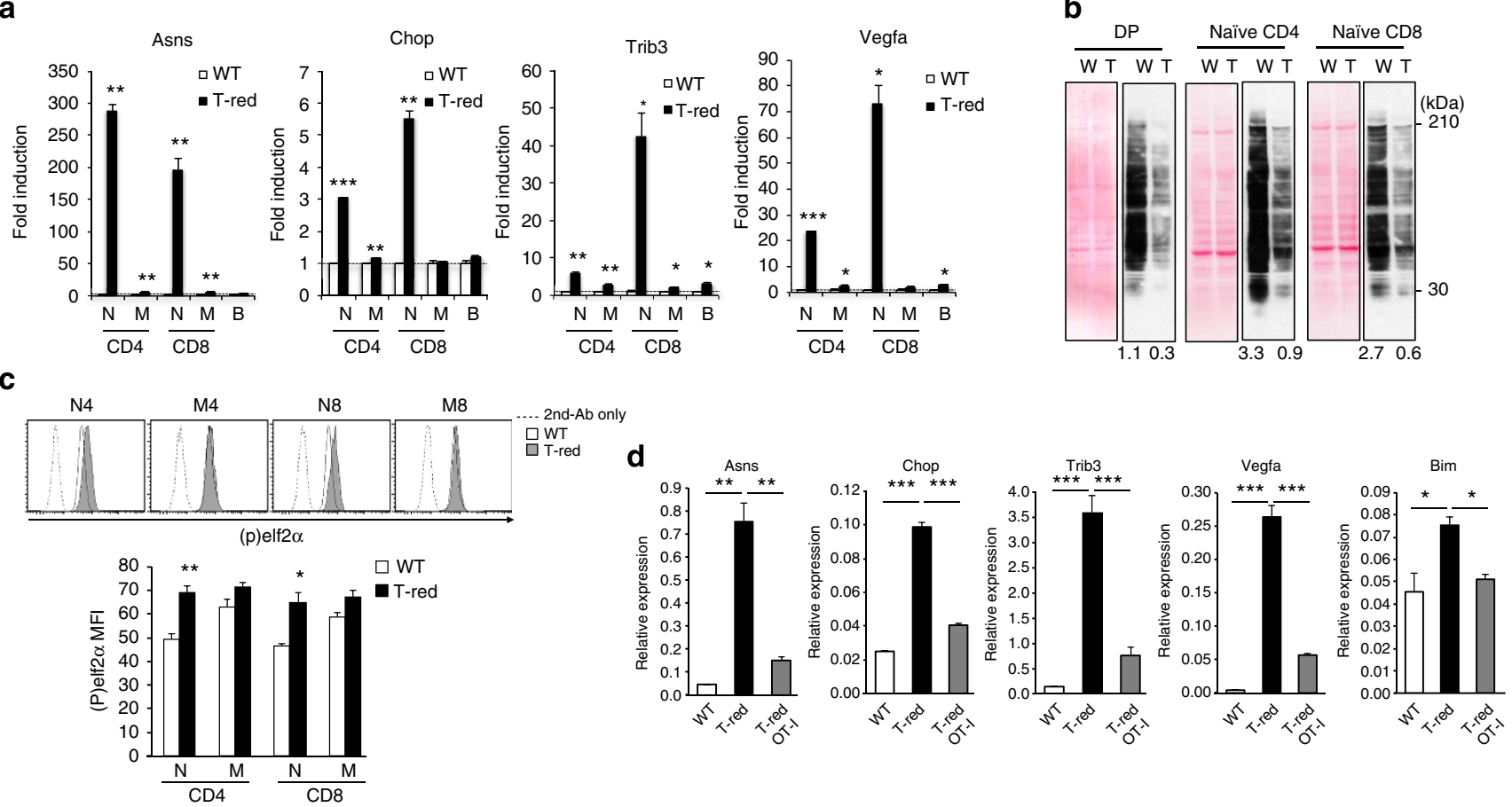

e

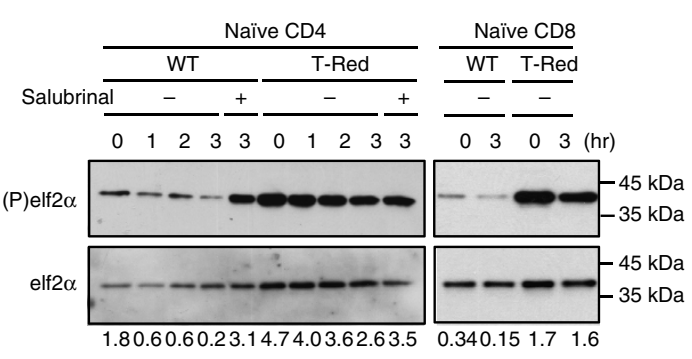

h
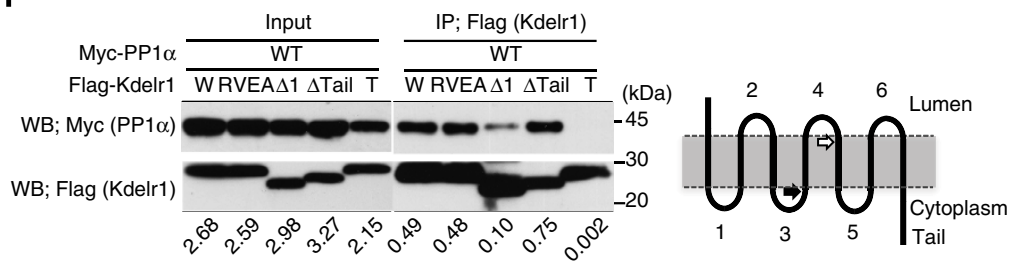

i
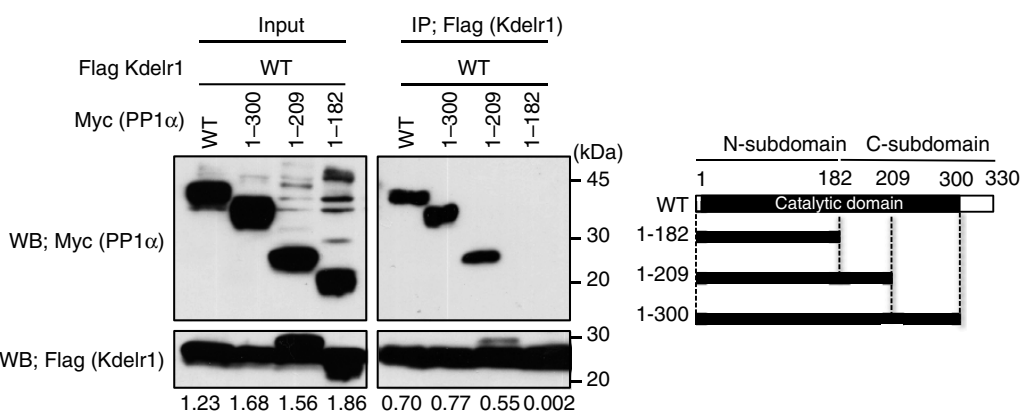

$\mathbf{f}$

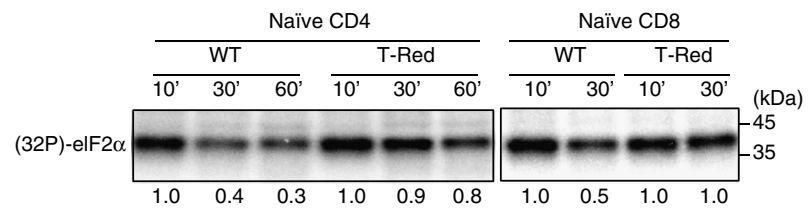

g

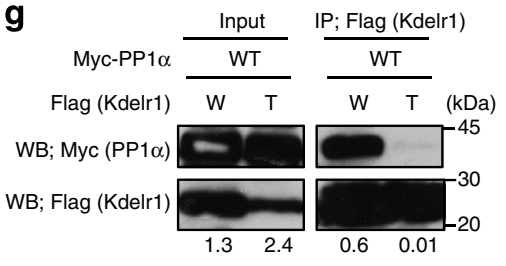

j

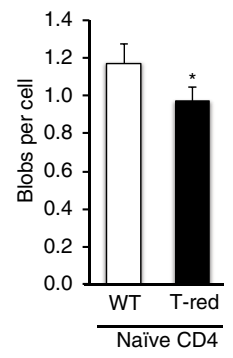


reported that a longer presence of DP thymocytes corresponds with more use of distal TCR $\alpha$ rearrangements ${ }^{46}$. In other words, DP thymocytes, which rapidly differentiate to the SP stage, elude apoptosis, which is enhanced in T-Red thymocytes. We hypothesize that the longer DP stage could be more stressful to induce ISR, just like in peripheral naïve $\mathrm{T}$ cells. Consistent with this notion, the number of DP thymocytes that have TCRrearrangements was reduced in T-Red mice compared with the number in WT mice (Fig. 1d). Moreover, an inducer of double strand breaks, etoposide, caused more death of T-Red DP thymocytes than that of WT thymocytes (Supplementary Fig. 8). Thus, it is possible that the shorter length of the DP thymocyte stage in T-Red mice may explain why proximal TCR $\alpha$ rearrangements are favoured in T-Red cells.

How the KDELR1-PP1 association regulates PP1 activity is a matter of future study. PP1 is known to have many regulatory proteins that determine phosphatase activity, substrate specificity and subcellular localization ${ }^{41}$, including the molecular chaperone $\mathrm{Bip}^{47,48}$, which has the KDEL motif and binds to KDELR1 (ref. 49). It was also reported that PP1/GADD34 complexes are localized to the $\mathrm{ER}^{50}$, where KDELR1 transports chaperones from Golgi compartments. We suggest then that KDELR1 may supply certain molecules, such as KDELR-binding chaperones that associate with PP1 and change the PP1 structure to dephosphorylate eIF $2 \alpha$ efficiently. Moreover, it is also possible that a strong TCR signalling might directly increase KDELR1 activity or reduce ISR response.

In summary, we generated an ENU mutant strain with a reduced number of naive $\mathrm{T}$ cells, which we named T-Red. Positional cloning and subsequent experiments identified the gene responsible for this effect as KDELR1. Mechanistic analysis suggested that KDELR1 regulates ISR in naïve $\mathrm{T}$ cells. Phosphorylation of eIF2 $\alpha$, a central checkpoint of ISR, is enhanced because activity of a key phosphatase of this pathway, PP1, is reduced in T-Red naïve T cells. T-Red KDELR1 did not associate with PP1, a property that correlated with reduced phosphatase activity. Indeed, naïv $\mathrm{T}$ cells in T-Red mice increased the expression of several target genes in the eIF $2 \alpha$ pathway, including Bim, Chop and Trib3, and caused apoptosis. We also hypothesize that physiological high-affinity TCR signal regulates T-Red KDELR1-mediated excess ISR in naïve T cells. We suggest that KDELR1 regulates ISR by controlling PP1 activity to maintain naïve T-cell homeostasis in vivo.

\footnotetext{
Methods

Mouse strains. C57BL/6 mice and $\mathrm{C} 3 \mathrm{H} / \mathrm{He}$ mice were purchased from Charles River Japan. All mice were maintained under specific pathogen-free conditions
}

according to the protocols of Osaka University Medical School and RIKEN Research Center for Allergy and Immunology (RCAI). Mice of both sexes were used. The age of mice is indicated in figure legends. There are no sample exclusion criteria. No randomization or blinding was used. Sample size of more than three mice was chosen to ensure power for Student's $t$-test unless the availability of mice was limited. All animal experiments were performed following the guidelines of the Institutional Animal Care and Use Committees of the Graduate School of Frontier Bioscience and Graduate School of Medicine, Osaka University; the Institute for Genetic Medicine and Graduate School of Medicine, Hokkaido University; and RIKEN RCAI.

Antibodies and reagents. The following antibodies were used for FACS staining at 200-fold dilution except for anti-CD90 antibodies, which were diluted to 2,000-fold: eFlour450-conjugated anti-CD4 (RM4-5), anti-CD8 (53-6.7) and anti-B220 (RA3-6B2) (eBioscience, San Diego, California); BV421-conjugated anti-CD19 (6D5) (BioLegend, San Diego); FITC-conjugated anti-CD44 (IM7) (eBioscience) and anti-IgD (11-26c.2a) (BD Biosciences, San Jose, California); PE-conjugated anti-CD25 (PC61), anti-CD45.2 (104), anti-CD62L (MEL14), anti-IL-17A (eBio17B7) (eBioscience), anti-IgM (R6-60.2) and anti-IgG1 (A85-1) (BD Biosciences); PE-Cy7-conjugated anti-CD3 (145-2C11) (BioLegend), anti-CD8 and anti-CD44 (eBioscience); APC-conjugated anti-CD4, anti-CD45.1 (A20), anti-CD90.1 (HIS51), anti-CD90.2 (53-2.1), anti-interferon (IFN)- $\gamma$ (XMG1.2) (eBioscience), and anti-CD19 (BioLegend); biotin-conjugated CD273 (TY25) (BioLegend); anti-Bim (Cell Signaling, Tokyo, Japan); and Alexa488-conjugated anti-rabbit IgG (Invitrogen, Tokyo). Antibodies for western blotting were as follows: anti-Bim and anti-phospho S51 eIF2 $\alpha$ (Cell Signaling); anti-FLAG M2 affinity gel and 3xFLAG peptide (Sigma, Tokyo); and anti-eIF2 $\alpha$, anti-Actin, anti-PP1 and HRP-conjugated anti-cMyc (Santa Cruz, Dallas, Texas). They were used at 100 -fold dilution.

Flow cytometry and cell sorting. For cell surface labelling, $\sim 10^{6}$ cells were incubated with fluorescence-conjugated antibodies for $30 \mathrm{~min}$ on ice in the presence of non-labelled anti-CD16/32 (2.4G2) antibody for blocking. Intracellular staining was performed using the Cytofix/Cytoperm kit (BD Biosciences), or the Foxp3 Fixation/Permeabilization kit (eBioscience) for phosphorylated eIF2 $\alpha$ staining. Cells were then analysed with the CyAn flow cytometer (Beckman Coulter, Tokyo). Collected data were analysed using Flowjo software (Tree Star, Ashland, Oregon). To purify naïve and memory T cells, splenocytes and lymph node cells were sorted based on their CD44 expression levels using the Moflo cell sorter (Beckman Coulter). $\mathrm{CD} 4{ }^{+} \mathrm{CD} 8{ }^{+} \mathrm{CD} 25^{-}$thymocytes were sorted as DP thymocytes. $\operatorname{IgM}^{+} \mathrm{CD} 273^{-} \mathrm{B}$ cells were sorted as naive $\operatorname{IgM}^{+} \mathrm{B}$ cells ${ }^{51,52}$. Cell purity was routinely $>98 \%$. Antibody dilutions were 100 -fold and 200 -fold for intracellular staining and cell sorting, respectively.

Establishment of ENU mutant mice and SNP analysis. ENU (Sigma) was injected intraperitoneally (i.p.) into B6 male mice twice at 1-week interval ${ }^{53}$. After a sterile period (10 to 11 weeks), these G0 male mice were mated with female B6 mice to generate G1 populations. G2 populations were made by artificial insemination using G1 sperm and normal B6 eggs. G3 offspring were generated by G2 intercrosses. About 30 G3 mice per G1 pedigree were checked for T-cell populations in peripheral blood. A mutant mouse was crossed with WT B6 to establish a mutant mouse line. For linkage analysis, T-Red mice (B6 background) were crossed with $\mathrm{C} 3 \mathrm{H} / \mathrm{HeJ}$ mice, and $\mathrm{F} 1$ mice were intercrossed. $\mathrm{F} 2$ populations were checked for CD44 levels on CD8 T cells in peripheral blood. The region responsible for the T-Red phenotype was determined by SNP analysis using tail DNA from F2 mice with high CD44 levels in T cells.

Figure 6 | ISR are enhanced in T-Red naïve T cells due to a functional defect of PP1. (a) ISR target genes in T-cell subsets and B cells were examined by qPCR. N, M and B indicate naïve, memory and B cells, respectively. Expression levels of WT populations were normalized as 1. Data represent the mean + s.d. $(n=2)$. (b) Global translation in DP thymocytes (DP), naïve T cells from WT (W) or T-Red (T) mice was examined by in vivo puromycin labelling (right). Ponceau-S staining is shown on the left. Numbers below the blots represent the intensity ratio of puromycin/Ponceau-S

(c) Phosphorylated-elF2 $\alpha$ in T-cell subsets were examined by flow cytometry. Representative FACS histograms from three independent experiments (top) and mean fluorescence intensity (MFl; bottom) are shown. Data represent the mean + s.e.m. $(n=3)$. (d) ISR target genes in naïve CD8 T cells from WT, T-Red or OT-I/T-Red mice were examined. Relative expressions to Hprt levels are shown. Data represent the mean + s.d. $(n=2)$. One-way ANOVA with post hoc Dunnett's test was used. (e) Naïve T cells were cultured and examined for phosphorylated-elF2 $\alpha$ levels. In some cultures, PP1/GADD34 inhibitor salubrinal was added. (f) Radiolabelled, phosphorylated-elF2 $\alpha$ was incubated for the indicated times with cell lysates from WT or T-Red naïve T cells, followed by SDS-PAGE and phosphor imaging. The intensity at $10 \mathrm{~min}$ in each group was normalized as 1.0. (g) Flag-KDELR1 WT or T-Red mutants and Myc-WT PP1 $\alpha$ were overexpressed in HEK293T cells and immunoprecipitated with anti-Flag beads, followed by blotting with anti-Flag or anti-Myc. (h) Association of KDELR1 mutants with WT PP1 $\alpha$. A schema of KDELR1 is shown to the right. Closed and open arrows indicate the location of RVEA and T-Red mutations, respectively. (i) Association of PP1 $\alpha$ mutants with WT KDELR1. A schema of PP1 $\alpha$ is shown to the right. Numbers below the blots in $\mathbf{g}-\mathbf{i}$ represent the ratio of PP1 $\alpha / K D E L R 1$. (j) Association of KDELR1 and PP1 $\alpha$ in naïve T cells examined by proximity ligation assay (PLA). Numbers of association signals per cell are shown. Data represent the mean + s.d. Representative images from more than three independent experiments are shown in b,d,f-h and i. Mice between 7 and 13 weeks old were used. ${ }^{\star} P<0.05$; ${ }^{\star \star} P<0.01 ;{ }^{\star \star \star} P<0.001$. 
Retroviral transduction of Kdelr1. B6 or T-Red mice were injected i.p. with $150 \mathrm{mg} \mathrm{kg}^{-1}$ of 5 -fluorouracil (Sigma, Tokyo) 3 to 5 days before harvesting bone marrow cells. Bone marrow cells were cultured for 2 days in the presence of $100 \mathrm{ng} \mathrm{ml}^{-1} \mathrm{IL}-6,100 \mathrm{ng} \mathrm{ml}^{-1}$ stem cell factor and $1 \%$ IL-3-conditioned medium. Phoenix cells were transfected with pMSCV-IRES-GFP KDELR1 or mock vectors, and virus-containing medium was collected after 2 days. Spin infection was performed twice in the presence of $4 \mathrm{mg} \mathrm{ml}^{-1}$ polybrene (Sigma). The resulting transduced bone marrow cells were transplanted into 9.5 Gy-irradiated B6 or T-Red mice. Recipient mice were analysed 8-12 weeks later.

Establishment of Kdelr1-deficient mice. KDELR1 conditional knockout mice were generated by a conventional homologous recombination technique in ES cells. The targeting vector was constructed in a pEZ-FRT-Lox-DT vector so that the second and third exons of the Kdelr1 gene were flanked with loxp sites (Supplementary Fig. 9). The FRT-site flanked neomycin-resistant gene was removed by crossing with flippase transgenic $(\mathrm{Tg})$ mice. Kdelr $1^{\text {flox }}{ }^{\text {flox }}$ mice were then crossed with CAG-cre Tg or CD4-cre Tg mice to generate conditional Kdelr1 knockout mice.

In vivo experiments. Collagen-induced arthritis was induced using a Mycobacterium bovis Bacillus Calmette-Guérin cell wall skeleton (BCG-CWS) emulsified in $\mathrm{CFA}^{54}$. Chicken type II collagen (Sigma Aldrich) was injected with CFA/BCG-CWS at $200 \mu \mathrm{g}$ per mouse at tail base on days 0 and 21. Serum IL-17 was measured using an IL-17 ELISA (enzyme-linked immunosorbent assay) kit (eBioscience). The anti-OVA response was elicited by i.p. injection of alumprecipitated OVA on days 0 and 5. Serum anti-OVA IgG1 and IgM levels were measured by ELISA in which an OVA-coated microtiter plate and alkaline phosphatase-conjugated anti-mouse IgG1 or IgM antibodies (Jackson Immunoresearch, Philadelphia, Pennsylvania) were used. T-cell responses to male-specific antigens were induced by injection of live splenocytes from male B6 mice $\left(2 \times 10^{7}\right.$ cells $)$ into congenic female B6 mice $\left(\mathrm{CD} 45.1^{+}\right)^{55}$. The donor $\mathrm{CD} 45.2^{+}$population in peripheral blood was examined over time. The detection limit was determined using anti-CD45.2 staining of blood leukocytes from B6.SJL mice. Bacterial infection using Listeria monocytogenes expressing OVA (LM-OVA) was performed as described ${ }^{56}$. In brief, mice were infected intravenously with 3,000 colony-forming units LM-OVA on day 0 . In some experiments, congenically marked recipients received 10,000 to 20,000 WT or T-Red OT-I cells 1 day before infection. On day 7, the OT-I population was checked by flow cytometry. The antigen-specific IFN- $\gamma$-secreting CD8 + T-cell population was determined by in vitro stimulation of splenocytes with OVA peptide (N4, SIINFEKL) for $4 \mathrm{~h}$ followed by intracellular IFN- $\gamma$ staining. Mixed bone marrow chimera mice were prepared by transplanting a mixture of T-cell-depleted bone marrow cells from WT (CD45.2/CD90.1), T-Red (CD45.1/CD45.2/CD90.2), OT-I (CD45.2/CD90.1/CD90.2) and T-Red/OT-I (CD45.2/CD90.2) mice into lethally irradiated (10 Gy) WT congenic mice (CD45.1/CD90.2). The ratio of the mixture was 4:4:1:1 to conform with a polyclonal situation. For in vivo survival assay of naïve $\mathrm{T}$ cells, equal numbers of sorted naïve $\mathrm{CD} 4$ or naïve $\mathrm{CD} 8 \mathrm{~T}$ cells from WT and Kdelr1 flox;ERT2-cre mice were mixed and transferred into congenic mice on day 0 . Tamoxyfen ( $1.5 \mathrm{mg}$ per mouse, p.o.) was administered on days 1,3 and 8 . The frequency of donor cells in blood was examined on days 1, 14 and 21 after donor cell transfer, and the absolute numbers of donor cells in the spleen were examined on day 21.

Metabolic labelling using puromycin. A non-radioactive method was employed to monitor protein synthesis ${ }^{57,58}$. In brief, mice were injected i.p. with puromycin (Sigma) at $20 \mathrm{mg} \mathrm{kg}^{-1}$. After $1 \mathrm{~h}$, splenocytes and thymocytes were harvested and sorted for naïv T cells and DP thymocytes. Total cell lysate was subjected to western blotting using anti-puromycin antibody at 8,000-fold dilution (clone 4G11, Millipore, Billerica, MA), followed by Ponceau-S staining of the membrane. Densitometry analysis was performed using ImageJ software (National Institutes of Health, Bethesda, Maryland).

In vitro T-cell culture. Sorted naïve or memory T cells $\left(1 \times 10^{5}\right.$ cells $)$ were cultured in a 96-well plate in the presence or absence of $2.5 \mathrm{ng} \mathrm{ml}^{-1}$ recombinant mouse IL-7 (Peprotech, Rocky Hill, New Jersey). Cells were stained with 7-AAD (eBioscience) and analysed by a CyAn flow cytometer (Beckman Coulter). The living cell population was determined as 7-AAD negative. In some experiments, naïe $\mathrm{T}$ cells or DP thymocytes were irradiated at $0.5 \mathrm{~Gy}$ or cultured with etoposide (Sigma) at $1 \mu \mathrm{g} \mathrm{ml}^{-1}$. For detection of eIF2 $\alpha$, sorted T cells that were freshly isolated or preincubated at $37^{\circ} \mathrm{C}$ for $1-2 \mathrm{~h}$ to reduce the endogenous level of eIF2 $\alpha$ phosphorylation were lysed in RIPA buffer, and total cell lysate was subjected to Western blotting. In some experiments, salubrinal (Santa Cruz), a PP1/GADD34 specific inhibitor, was added at $10 \mu \mathrm{M}$ after the preincubation. For Th1 or Th17 cell differentiation, sorted naïve $\mathrm{CD} 4 \mathrm{~T}$ cells were cultured for 4-5 days with bone marrow-derived dendritic cells and soluble anti-CD3 $\varepsilon$ mAb (145-2C11) in the presence of IL-12 (for Th1) or IL-6 and TGF- $\beta$ (for Th17). Intracellular cytokine staining was performed using fluorescence-labelled anti-IFN- $\gamma$ and anti-IL-17A at 200 -fold dilutions (eBioscience).
Real-time PCRs. The 7,300 real-time PCR system (ABI, Tokyo) and SYBR FAST PCR Mix or PROBE FAST PCR Mix (KAPA Biosystems, Woburn) were used to quantify the expression levels of each gene and HPRT mRNA. Total RNA was prepared from cell-sorted, purified T cells using the GenElute Mammalian total RNA kit (Sigma). The conditions for real-time PCR were 40 cycles at $94^{\circ} \mathrm{C}$ for $15 \mathrm{~s}$ followed by $60^{\circ} \mathrm{C}$ for $60 \mathrm{~s}$ (SYBR green), or 40 cycles at $94^{\circ} \mathrm{C}$ for $3 \mathrm{~s}$, followed by $60^{\circ} \mathrm{C}$ for $30 \mathrm{~s}$ (FAM and TAMRA dual-labelled probes (Sigma)). Relative mRNA expression levels were normalized to the levels of Hprt mRNA. qPCR primer sequences are: Bim, 5'FAM-TGAACTCGTCTCCGATCCGCCGCA-TAMRA3', $5^{\prime}$-ACGACAGTCTCAGGAGGAACC- $3^{\prime}$ and $5^{\prime}$-CGGTAATCATTTGCAAACA CCCTC-3'; Chop, 5'FAM-TCTTGACCCTGCGTCCCTAGCTTGGC-TAMRA3', $5^{\prime}$-CCCAGGAAACGAAGAGGAAGAA- $3^{\prime}$ and $5^{\prime}$-GGGATGTGCGTGTGAC CTC- ${ }^{\prime}$; Hprt, 5'FAM-ATCCAACAAAGTCTGGCCTGTATCCAACAC-TAMRA $3^{\prime}$, $5^{\prime}$-AGCCCCAAAATGGTTAAGGTTG- $3^{\prime}$ and $5^{\prime}$-CAAGGGCATATCCAACAA CAAAC- $3^{\prime}$; Asns, $5^{\prime}$-GGCCCTGGATGAAGTCATATT- $3^{\prime}$ and $5^{\prime}$-CACCACGC TGTCTGTGTTCT- $3^{\prime}$; Vegfa, $5^{\prime}$-TCACCAAAGCCAGCACATAG- $3^{\prime}$ and $5^{\prime}$-AAT GCTTTCTCCGCTCTGAA- $3^{\prime}$; and Trib3, $5^{\prime}$-GCCTTATATCCTTTTGGAA CGA- $3^{\prime}$ and $5^{\prime}$-AGATGTAAAGGAGCCGAGAGC- $3^{\prime}$.

Immunoprecipitation and western blotting. HEK293T cells were transfected with a calcium phosphate transfection method. The pBOS-EF expression vector with Myc or FLAG tag was used for forced expression. Total cell lysate was immunoprecipitated with anti-FLAG M2 affinity gel (Sigma, Tokyo). The $3 \times$ FLAG peptide (Sigma) was used to elute the immunoprecipitated fraction. SDS-polyacrylamide gel electrophoresis (SDS-PAGE) and western blotting was performed by standard methods ${ }^{27}$. Densitometry analysis was performed using ImageJ software. The original gel images are shown in Supplementary Fig. 10.

TCR J $\alpha$ and TCR $\beta$ usage/recombination. Total RNA was extracted from DP thymocytes, and CDNA was synthesized using oligo dT. TCR V $\alpha 8$-C $\alpha$ fragments were amplified by PCR and blotted with ${ }^{32}$ P-labelled J $\alpha$ specific oligonucleotide probes ${ }^{59}$. Hybridization was performed overnight at $50^{\circ} \mathrm{C}$, and washing was carried out for $20 \mathrm{~min}$ at $60^{\circ} \mathrm{C}$ twice in $6 \times \mathrm{SSC} / 0.1 \%$ SDS. Primer and probe sequences are shown in Supplementary Table 1. TCR V $\beta$ usage was examined by flow cytometry using the V $\beta$ TCR screening panel (BD Biosciences). $C \beta 1 \mathrm{mRNA}$ levels were measured by qPCR using SYBR green. TCR D $\beta 2$-J $\beta 2$ recombination was examined by genomic DNA PCR using primers listed in Supplementary Table 1. The insulin gene was used as a positive control for genomic DNA amplification $^{60}$.

Dephosphorylation assay of elF2 $\alpha$. A dephosphorylation assay for eIF $2 \alpha^{61,62}$ was performed using naïve T-cell lysates. Sorted naïve T cells were lysed in a cell lysis buffer containing $20 \mathrm{mM}$ Tris- $\mathrm{HCl}, \mathrm{pH} 7.4,0.5 \%$ Triton-X-100, $50 \mathrm{mM} \mathrm{NaCl}$, $10 \%$ glycerol and $0.1 \mathrm{mM}$ EDTA. Recombinant GST-PERK and His-eIF2 $\alpha$ (Sigma) were incubated for $30 \mathrm{~min}$ at $30^{\circ} \mathrm{C}$ in the presence of $5 \mu \mathrm{Ci}\left[\gamma^{-32} \mathrm{P}\right] \mathrm{ATP}$. After removal of unincorporated isotopes, a portion of the radiolabeled proteins was incubated at $30^{\circ} \mathrm{C}$ with $5 \mathrm{ml}$ of total T-cell lysate in a $10-\mu l$ reaction (dephosphorylation buffer; $20 \mathrm{mM}$ Tris- $\mathrm{HCl}$, pH 7.4, $50 \mathrm{mM} \mathrm{KCl}, 2 \mathrm{mM} \mathrm{MgCl}_{2}$, $0.1 \mathrm{mM}$ EDTA, $0.8 \mathrm{mM}$ ATP). The reaction was stopped by adding $5 \times$ SDS-PAGE sample buffer followed by boiling and SDS-PAGE. Images were obtained using Typhoon Phosphorimager (GE Healthcare, Tokyo). Densitometry analysis was performed using ImageJ software.

Measurement of mitochondria stress responses. Mitochondrial membrane depolarization was examined using the MitoPT JC-1 kit (ImmunoChemistry Technologies, Bloomington, Minnesota). Total thymocytes were stained with JC-1 dye followed by cell surface staining. A shift of fluorescence was detected by flow cytometry. As a positive control of the JC-1 staining, thymocytes were incubated with carbonylcyanide $m$-chlorophenylhydrazone at $50 \mu \mathrm{M}$ for $30 \mathrm{~min}$. The expressions of Clpp and $m t H S P 60$, two target genes for mitochondrial stress ${ }^{33,34}$, were examined by real-time PCR.

Measurement of ATP/ROS/iron levels. Intracellular ATP levels were measured using a commercially available luciferase-based kit (Toyo B-Net, Tokyo). For ROS detection, splenocytes were stained with $2 \mu \mathrm{M} \mathrm{H}_{2} \mathrm{D}$-CFDA for $15 \mathrm{~min}$ at $37^{\circ} \mathrm{C}$ followed by cell surface staining for flow cytometry. Iron levels were examined using calcein-AM (Sigma $)^{63}$. Splenocytes were stained with $0.025 \mu \mathrm{M}$ calcein-AM for $30 \mathrm{~min}$ at $37^{\circ} \mathrm{C}$ followed by cell surface staining for flow cytometry. Binding of intracellular iron to calcein quenched the calcein fluorescence.

Proximity ligation assay. PLA was performed to examine KDELR1 and PP1 association at endogenous expression levels. Sorted naïve T cells from WT and T-Red mice were seeded in a microscopy chamber (Ibidi), then fixed and permeabilized using the Cytofix/Cytoperm kit (BD Biosciences). After incubation with anti-KDELR1 and anti-PP1 (Santa Cruz) at 100-fold dilutions, a PLA reaction was performed using a commercially available kit (Duolink, Sigma). Blob-like fluorescent PLA signals were obtained by Z-stack imaging using confocal 
microscopy. The number of blobs was automatically counted in more than 200 cells by computer software.

Statistical analysis. Student's $t$-test (two-tailed) was used for the statistical analysis of differences between two groups unless stated otherwise. For multiple comparisons, one-way ANOVA (analysis of variance) and post hoc Dunnett's test were used. A paired Student's $t$-test was used for some experiments. Wilcoxon test was used for the evaluation of arthritis clinical scores. $P$ values $<0.05$ were considered statistically significant.

\section{References}

1. Pelham, H. R. B. The dynamic organisation of the secretory pathway. Cell Struct. Funct. 21, 413-419 (1996).

2. Ellgaard, L., Molinari, M. \& Helenius, A. Setting the standards: quality control in the secretory pathway. Science 286, 1882-1888 (1999).

3. Lewis, M. J. \& Pelham, H. R. A human homologue of the yeast HDEL receptor. Nature 348, 162-163 (1990).

4. Semenza, J. C., Hardwick, K. G., Dean, N. \& Pelham, H. R. ERD2, a yeast gene required for the receptor-mediated retrieval of luminal ER proteins from the secretory pathway. Cell 61, 1349-1357 (1990).

5. Yamamoto, K. et al. The KDEL receptor modulates the endoplasmic reticulum stress response through mitogen-activated protein kinase signaling cascades. J. Biol. Chem. 278, 34525-34532 (2003).

6. Pulvirenti, T. et al. A traffic-activated Golgi-based signalling circuit coordinates the secretory pathway. Nat. Cell Biol. 10, 912-922 (2008).

7. Capitani, M. \& Sallese, M. The KDEL receptor: new functions for an old protein. FEBS Lett. 583, 3863-3871 (2009).

8. Williams, B. R. PKR; a sentinel kinase for cellular stress. Oncogene 18, 6112-6120 (1999)

9. Harding, H. P. et al. Regulated translation initiation controls stress-induced gene expression in mammalian cells. Mol. Cell 6, 1099-1108 (2000).

10. Lu, L., Han, A. P. \& Chen, J. J. Translation initiation control by heme-regulated eukaryotic initiation factor 2alpha kinase in erythroid cells under cytoplasmic stresses. Mol. Cell. Biol. 21, 7971-7980 (2001).

11. Harding, H. P., Calfon, M., Urano, F., Novoa, I. \& Ron, D. Transcriptional and translational control in the mammalian unfolded protein response. Annu. Rev. Cell Dev. Biol. 18, 575-599 (2002).

12. Donze, O. et al. The protein kinase PKR: a molecular clock that sequentially activates survival and death programs. EMBO J. 23, 564-571 (2004).

13. Moreno, J. A. et al. Sustained translational repression by eIF2alpha-P mediates prion neurodegeneration. Nature 485, 507-511 (2012).

14. Harding, H. P. et al. Ppp1r15 gene knockout reveals an essential role for translation initiation factor 2 alpha (eIF2alpha) dephosphorylation in mammalian development. Proc. Natl Acad. Sci. USA 106, 1832-1837 (2009).

15. Aridor, M. \& Balch, W. E. Integration of endoplasmic reticulum signaling in health and disease. Nat. Med. 5, 745-751 (1999).

16. Zhang, P. et al. The PERK eukaryotic initiation factor 2 alpha kinase is required for the development of the skeletal system, postnatal growth, and the function and viability of the pancreas. Mol. Cell. Biol. 22, 3864-3874 (2002).

17. Chang, R. C., Wong, A. K., Ng, H. K. \& Hugon, J. Phosphorylation of eukaryotic initiation factor-2alpha (eIF2alpha) is associated with neuronal degeneration in Alzheimer's disease. Neuroreport 13, 2429-2432 (2002).

18. Scheu, S. et al. Activation of the integrated stress response during $\mathrm{T}$ helper cell differentiation. Nat. Immunol. 7, 644-651 (2006).

19. Kamimura, D. \& Bevan, M. J. Endoplasmic reticulum stress regulator XBP-1 contributes to effector CD8 $+\mathrm{T}$ cell differentiation during acute infection. J Immunol. 181, 5433-5441 (2008).

20. Surh, C. D. \& Sprent, J. Homeostatic T cell proliferation: how far can T cells be activated to self-ligands? J Exp Med. 192, F9-F14 (2000).

21. Bouillet, P. et al. Proapoptotic Bcl-2 relative Bim required for certain apoptotic responses, leukocyte homeostasis, and to preclude autoimmunity. Science 286, 1735-1738 (1999).

22. Hildeman, D. A. et al. Activated T cell death in vivo mediated by proapoptotic bcl-2 family member bim. Immunity 16, 759-767 (2002).

23. Marrack, P. \& Kappler, J. Control of T cell viability. Annu. Rev. Immunol. 22, 765-787 (2004).

24. Wojciechowski, S. et al. $\mathrm{Bim} / \mathrm{Bcl}-2$ balance is critical for maintaining naive and memory T cell homeostasis. J. Exp. Med. 204, 1665-1675 (2007).

25. Puthalakath, H. et al. ER stress triggers apoptosis by activating BH3-only protein Bim. Cell 129, 1337-1349 (2007).

26. Hitotsumachi, S., Carpenter, D. A. \& Russell, W. L. Dose-repetition increases the mutagenic effectiveness of $\mathrm{N}$-ethyl-N-nitrosourea in mouse spermatogonia. Proc. Natl Acad. Sci. USA 82, 6619-6621 (1985).

27. Sawa, S. et al. Autoimmune arthritis associated with mutated interleukin (IL)-6 receptor gp130 is driven by STAT3/IL-7-dependent homeostatic proliferation of CD4+ T cells. J. Exp. Med. 12, 1459-1470 (2006).
28. Ueda, N. et al. CD1d-restricted NKT cell activation enhanced homeostatic proliferation of CD8 $+\mathrm{T}$ cells in a manner dependent on IL-4. Int. Immunol. 18, 1397-1404 (2006).

29. Krangel, M. S., Carabana, J., Abbarategui, I., Schlimgen, R. \& Hawwari, A. Enforcing order within a complex locus: current perspectives on the control of $\mathrm{V}(\mathrm{D}) \mathrm{J}$ recombination at the murine T-cell receptor alpha/delta locus. Immunol. Rev. 200, 224-232 (2004).

30. Jackson, A. M. \& Krangel, M. S. Turning T-cell receptor beta recombination on and off: more questions than answers. Immunol. Rev. 209, 129-141 (2006).

31. Hildeman, D., Jorgensen, T., Kappler, J. \& Marrack, P. Apoptosis and the homeostatic control of immune responses. Curr. Opin. Immunol. 19, 516-521 (2007).

32. Kurtulus, S. et al. Bcl-2 allows effector and memory CD8 + T cells to tolerate higher expression of Bim. J. Immunol. 186, 5729-5737 (2011).

33. Zhao, Q. et al. A mitochondrial specific stress response in mammalian cells. EMBO J. 21, 4411-4419 (2002)

34. Nargund, A. M., Pellegrino, M. W., Fiorese, C. J., Baker, B. M. \& Haynes, C. M. Mitochondrial import efficiency of ATFS-1 regulates mitochondrial UPR activation. Science 337, 587-590 (2012).

35. Ito, T., Yang, M. \& May, W. S. RAX, a cellular activator for double-stranded RNA-dependent protein kinase during stress signaling. J. Biol. Chem. 274, 15427-15432 (1999)

36. Han, A. P. et al. Heme-regulated eIF2alpha kinase (HRI) is required for translational regulation and survival of erythroid precursors in iron deficiency. EMBO J. 20, 6909-6918 (2001).

37. Ioudina, M., Uemura, E. \& Greenlee, H. W. Glucose insufficiency alters neuronal viability and increases susceptibility to glutamate toxicity. Brain Res. 1004, 188-192 (2004).

38. Muaddi, H. et al. Phosphorylation of eIF2alpha at serine 51 is an important determinant of cell survival and adaptation to glucose deficiency. Mol. Biol. Cell. 21, 3220-3231 (2010)

39. Verfaillie, T. et al. PERK is required at the ER-mitochondrial contact sites to convey apoptosis after ROS-based ER stress. Cell Death Differ. 19, 1880-1891 (2012).

40. Boyce, M. et al. A selective inhibitor of eIF2alpha dephosphorylation protects cells from ER stress. Science 307, 935-939 (2005).

41. Bollen, M. Combinatorial control of protein phosphatase-1. Trends Biochem. Sci. 26, 426-431 (2001).

42. Egloff, M. P. et al. Structural basis for the recognition of regulatory subunits by the catalytic subunit of protein phosphatase 1. EMBO J. 16, 1876-1887 (1997).

43. Aoe, T. et al. The KDEL receptor, ERD2, regulates intracellular traffic by recruiting a GTPase-activating protein for ARF1. EMBO J. 16, 7305-7316 (1997).

44. Goldberg, J. et al. Three-dimensional structure of the catalytic subunit of protein serine/threonine phosphatase-1. Nature 376, 745-753 (1995).

45. Scheel, A. A. \& Pelham, H. R. Identification of amino acids in the binding pocket of the human KDEL receptor. J. Biol. Chem. 273, 2467-2472 (1998)

46. Guo, J. et al. Regulation of the TCRalpha repertoire by the survival window of CD4 $(+)$ CD8 $(+)$ thymocytes. Nat. Immunol. 3, 469-476 (2002).

47. Chun, Y. S. et al. Role of the $78-\mathrm{kDa}$ glucose-regulated protein as an activity modulator of protein phosphatase1gamma2. Biochem. Biophys. Res. Commun. 259, 300-304 (1999).

48. Garcia-Bonilla, L. et al. Regulatory proteins of eukaryotic initiation factor 2-alpha subunit (eIF2 alpha) phosphatase, under ischemic reperfusion and tolerance. J. Neurochem. 103, 1368-1380 (2007).

49. Munro, S. \& Pelham, H. R. A C-terminal signal prevents secretion of luminal ER proteins. Cell 48, 899-907 (1987).

50. Brush, M. H., Weiser, D. C. \& Shenolikar, S. Growth arrest and DNA damage-inducible protein GADD34 targets protein phosphatase 1 alpha to the endoplasmic reticulum and promotes dephosphorylation of the alpha subunit of eukaryotic translation initiation factor 2. Mol. Cell. Biol. 23, 1292-1303 (2003).

51. Good-Jacobson, K. L. et al. PD-1 regulates germinal center B cell survival and the formation and affinity of long-lived plasma cells. Nat. Immunol. 11, 535-542 (2010).

52. Kometani, K. et al. Repression of the transcription factor Bach2 contributes to predisposition of IgG1 memory B cells toward plasma cell differentiation. Immunity 39, 136-147 (2013).

53. Inoue, M. et al. A series of maturity onset diabetes of the young, type 2 (MODY2) mouse models generated by a large-scale ENU mutagenesis program. Hum. Mol. Genet. 13, 1147-1157 (2004).

54. Kitabayashi, C. et al. Zinc suppresses Th17 development via inhibition of STAT3 activation. Int. Immunol. 22, 375-386 (2010).

55. Tyznik, A. J. \& Bevan, M. J. The surprising kinetics of the T cell response to live antigenic cells. J. Immunol. 179, 4988-4995 (2007).

56. Kamimura, D. \& Bevan, M. J. Naive CD8 + T cells differentiate into protective memory-like cells after IL-2 anti IL-2 complex treatment in vivo. J. Exp. Med. 204, 1803-1812 (2007) 
57. Schmidt, E. K., Clavarino, G., Ceppi, M. \& Pierre, P. SUnSET, a nonradioactive method to monitor protein synthesis. Nat Methods. 6, 275-277 (2009).

58. Goodman, C. A. et al. Novel insights into the regulation of skeletal muscle protein synthesis as revealed by a new nonradioactive in vivo technique. FASEB J. 25, 1028-1039 (2011).

59. Hawwari, A., Bock, C. \& Krangel, M. S. Regulation of T cell receptor alpha gene assembly by a complex hierarchy of germline Jalpha promoters. Nat. Immunol. 6, 481-489 (2005)

60. Würch, A. et al. Requirement of CD3 complex-associated signaling functions for expression of rearranged T cell receptor beta VDJ genes in early thymic development. J. Exp. Med. 188, 166-1678 (1998).

61. Harding, H. P., Zhang, Y. \& Ron, D. Protein translation and folding are coupled by an endoplasmic-reticulum-resident kinase. Nature 397, 271-274 (1999).

62. Novoa, I., Zeng, H., Harding, H. P. \& Ron, D. Feedback inhibition of the unfolded protein response by GADD34-mediated dephosphorylation of eIF2alpha. J. Cell Biol. 153, 1011-1022 (2001).

63. Fonseca, A. M., Pereira, C. F., Porto, G. \& Arosa, F. A. Red blood cells upregulate cytoprotective proteins and the labile iron pool in dividing human $\mathrm{T}$ cells despite a reduction in oxidative stress. Free Radic. Biol. Med. 35, 1404-1416 (2003)

\section{Acknowledgements}

We thank Dr Lloyd Ruddock (University of Oulu, Finland) and Dr Michael J. Bevan (University of Washington, Seattle) for the Kdelr1 cDNA and Listeria-OVA, respectively. We also thank the excellent technical assistance and experimental help provided by

Ms Satoko Ojimi (RIKEN RCAI, Yokohama, Japan), Ms Yuko Ishikura (RIKEN RCAI, Yokohama, Japan), Ms Noriko Kumai (Hokkaido University, Sapporo, Japan), Mr Shunji Mitani (Osaka University, Osaka, Japan) and Chiemi Nakayama (Hokkaido University, Sapporo, Japan). We appreciate Ms Ryoko Masuda (Osaka University, Osaka, Japan) for her excellent secretarial assistance, and Dr Kazuhiro Iwai (Osaka University, Osaka,
Japan), Dr Otsura Niwa (Kyoto University, Kyoto, Japan) and Dr Peter Karagiannis (RIKEN QBiC, Osaka, Japan) for carefully reading the manuscript. This work was supported by KAKENHI (D.K., M.M. and T.H.), JSPS Postdoctoral Fellowship for Foreign Researchers (A.S.), the JST-CREST programme (M.M. and T.H.) and the Osaka Foundation for the Promotion of Clinical Immunology (M.M.).

\section{Author contributions}

M.M. conceived the project, supervised most experiments and wrote the manuscript. D.K. and K.K. performed most of the experiments and D.K. contributed to writing the manuscript. The other authors contributed to specific experiments and analyses.

\section{Additional information}

Supplementary Information accompanies this paper at http://www.nature.com/ naturecommunications

Competing financial interests: The authors declare no competing financial interests.

Reprints and permission information is available online at http://npg.nature.com/ reprintsandpermissions/

How to cite this article: Kamimura, D. et al. KDEL receptor 1 regulates T-cell homeostasis via PP1 that is a key phosphatase for ISR. Nat. Commun. 6:7474 doi: $10.1038 /$ ncomms8474 (2014).

\section{(c) (i)}

This work is licensed under a Creative Commons Attribution 4.0 International License. The images or other third party material in this article are included in the article's Creative Commons license, unless indicated otherwise in the credit line; if the material is not included under the Creative Commons license, users will need to obtain permission from the license holder to reproduce the material. To view a copy of this license, visit http://creativecommons.org/licenses/by/4.0/ 